UCLA $/ 08 / \mathrm{TEP} / 24$

SLAC-PUB-13361

\title{
Manifest Ultraviolet Behavior for the Three-Loop Four-Point Amplitude of $\mathcal{N}=8$ Supergravity
}

\author{
Z. $\operatorname{Bern}^{a}$, J. J. M. Carrasco ${ }^{a}$, L. J. Dixon ${ }^{b}$, H. Johansson ${ }^{a}$ and R. Roiban ${ }^{c}$ \\ ${ }^{a}$ Department of Physics and Astronomy, \\ UCLA, Los Angeles, CA 90095-1547, USA \\ ${ }^{b}$ Stanford Linear Accelerator Center, \\ Stanford University, Stanford, CA 94309, USA \\ ${ }^{c}$ Department of Physics, Pennsylvania State University, University Park, PA 16802, USA
}

(Dated: August, 2008)

\begin{abstract}
Using the method of maximal cuts, we obtain a form of the three-loop four-point scattering amplitude of $\mathcal{N}=8$ supergravity in which all ultraviolet cancellations are made manifest. The Feynman loop integrals that appear have a graphical representation with only cubic vertices, and numerator factors that are quadratic in the loop momenta, rather than quartic as in the previous form. This quadratic behavior reflects cancellations beyond those required for finiteness, and matches the quadratic behavior of the three-loop four-point scattering amplitude in $\mathcal{N}=4$ superYang-Mills theory. By direct integration we confirm that no additional cancellations remain in the $\mathcal{N}=8$ supergravity amplitude, thus demonstrating that the critical dimension in which the first ultraviolet divergence occurs at three loops is $D_{c}=6$. We also give the values of the threeloop divergences in $D=7,9,11$. In addition, we present the explicitly color-dressed three-loop four-point amplitude of $\mathcal{N}=4$ super-Yang-Mills theory.

PACS numbers: 04.65.+e, 11.15.Bt, 11.30.Pb, 11.55.Bq
\end{abstract}




\section{INTRODUCTION}

Recently, the widely held belief that it is impossible to construct a consistent fourdimensional point-like theory of quantum gravity has been called into question for maximally supersymmetric [1] $\mathcal{N}=8$ supergravity. In particular, an integral representation of the three-loop four-point amplitude [2] in this theory has been obtained explicitly, which exhibits cancellations beyond those needed for ultraviolet finiteness. Moreover, for a class of terms accessible by isolating one-loop subdiagrams via generalized unitarity [3], the oneloop "no-triangle" property [4, 5, 6, 7, 8] - recently proven in refs. [9, 10] - shows that at least a subset of these cancellations persist to all loop orders [11]. Interestingly, M theory and string theory have also been used to argue either for the finiteness of $\mathcal{N}=8$ supergravity [12], or that divergences are delayed through at least nine loops [13, 14], though issues with decoupling towers of massive states [15] may alter these conclusions. If a perturbatively ultraviolet-finite point-like theory of quantum gravity could be constructed, the underlying mechanism responsible for the required cancellations would have a profound impact on our understanding of gravity.

Over the years supersymmetry has been studied extensively as a mechanism for delaying the onset of divergences in gravity theories (see e.g. refs. [16, 17]). In particular, the existence, or conjectured existence, of various off-shell superspace formalisms restricts the form and dimensions of potential counterterms, leading to bounds on the first permissible loop order at which an ultraviolet divergence might appear in $\mathcal{N}=8$ supergravity. The precise bound depends on the detailed set of assumptions. For example, if an off-shell superspace with $\mathcal{N}=6$ supersymmetries manifest were to exist, potential $D=4$ divergences would be delayed to at least five loops [17], while the existence of a superspace with $\mathcal{N}=7$ supersymmetries manifest would delay the first potential divergence to at least six loops [17]. Similarly, if one were to assume the existence of a fully covariant off-shell superspace with $\mathcal{N}=8$ supersymmetries manifest, then the first potential divergence would be pushed to the seven loop order [18]. Full superspace invariants, which could act as potential counterterms, have been constructed at eight loops, suggesting that a divergence might appear at this loop order, if it does not appear earlier [19]. This first potential divergence can even be pushed to the nine loop order, with an additional speculative assumption that all fields respect ten-dimensional general coordinate invariance [20]. This bound coincides with the 
one argued [14] from the type II string theory non-renormalization theorem of Berkovits [13]. Beyond this order, no purely supersymmetric mechanism has been suggested for preventing the onset of divergences. In fact, on dimensional grounds one can argue that, for any supergravity theory to be ultraviolet finite to all loop orders, novel cancellations beyond the known supersymmetric ones must exist.

Surprisingly, cancellations beyond those implied by naive loop-momentum powercounting appear to be generic in gravity theories, as suggested by the one-loop study of ref. [21]. This reference demonstrated that these novel one-loop cancellations are directly connected to the remarkably good high-energy behavior of gravity tree amplitudes under the complex deformations used to prove on-shell recursion relations in gravity [10, 22, 23, 24, 25, 26]. Recently [27, 28], these tree-level properties have been understood in terms of a space-like gauge similar to light-cone gauge [29], where an enhanced Lorentz symmetry was shown to exist under large complex deformations.

For non-supersymmetric theories, these cancellations are insufficient to render the theory ultraviolet finite. Indeed, it is a classical result that gravity coupled to matter generically diverges at one loop [30, 31, 32]. Pure Einstein gravity does not possess a viable counterterm at one loop, delaying the divergence to two loops [30, 33, 34]. The presence of a twoloop divergence in pure Einstein gravity was established by Goroff and Sagnotti and by van de Ven, through direct computation [35, 36].

Supersymmetric cancellations can often act on top of any generic cancellations. In particular, in the case of $\mathcal{N}=8$ supergravity at one loop, such cancellations combine to cause the vanishing of the coefficients of all scalar bubble and triangle integrals [4, 5, 6, 7, 8], proofs of which have been given recently [9, 10]. The one-loop amplitudes can be expressed solely in terms of box integrals, multiplied by rational coefficients, exactly as is the case for $\mathcal{N}=4$ super-Yang-Mills theory [37]. This one-loop no-triangle property can be used, via unitarity, to understand a class of higher-loop cancellations [11]. However, it does not account for all of them, because regions where two or more overlapping loop momenta become large are not covered directly by the one-loop analysis. More generally, the picture that emerges at higher loops is that the excellent ultraviolet behavior found in explicit calculations [2, 38] is due to a combination of generic cancellations with supersymmetric ones [11, 21]. Interestingly, the absence of bubble integrals in theories with $\mathcal{N} \geq 5$ supersymmetry at one loop might lead one to speculate that such theories may also be finite, if $\mathcal{N}=8$ supergravity is finite [21]. 
To establish the critical dimension where divergences first occur at a given loop order, we evaluate amplitudes directly. A general strategy for obtaining loop amplitudes in gravity theories was first given in ref. [38], following earlier work in $\mathcal{N}=4$ super-Yang-Mills theory at one loop [37] and higher loops [39]. Using generalized unitarity, at any loop order we can evaluate scattering amplitudes from products of on-shell tree amplitudes [3]. In gravity theories we can then exploit the Kawai-Lewellen-Tye (KLT) relations [40], which express gravity tree amplitudes directly in terms of gauge theory tree amplitudes. (Subsequent generalizations and other approaches to these relations may be found in refs. [41, 42, 43, 44].) The net effect is that we can map complicated gravity calculations into substantially simpler gauge theory calculations. This simplification is especially important when evaluating generalized unitarity cuts in $D$ dimensions.

In ref. [2] this strategy was used to obtain the complete three-loop four-point amplitude of $\mathcal{N}=8$ supergravity. The result of this calculation explicitly demonstrated hidden cancellations beyond those identified in the earlier partial calculation of ref. [38]. However, the cancellations took place between different terms of the integral representation of ref. [2]. Each of the nine contributing "parent" integrals had a graphical representation with only cubic vertices, and numerator factors that were quartic in the loop momenta. (In general, a parent integral refers to an integral with the maximal number of propagators allowed in a given amplitude. The graphs for such integrals have only cubic vertices.) One of the main purposes of the present paper is to provide an improved form of the amplitude in which the true ultraviolet behavior is manifest. The new form can be written in terms of the same nine parent integrals, but terms have been shuffled between the different integrals, so that each numerator factor is now quadratic in the loop momenta. In this way, no term in the amplitude has a worse ultraviolet behavior than does the sum over all terms.

The new representation of the three-loop four-point amplitude is constructed using the technique of maximal cuts, developed in ref. [45]. This technique uses generalized unitarity [3], starting with the maximum number of cut propagators and systematically reducing the number of cut propagators, in order to construct the complete amplitude. As observed by Britto, Cachazo and Feng [46], on-shell massless three-point amplitudes can be defined by analytically continuing momenta to complex values [35, 47]. Maximal cuts involve products of three-point tree amplitudes alone, and are the simplest cuts to evaluate. Near-maximal cuts, in which one or two of the maximal-cut propagators have been allowed to go off-shell, 
are the next simplest to consider, and so on. We use the near maximal cuts to fix contact terms that may have been missed by the purely maximal ones. The maximal-cut technique, by removing cut conditions one by one from the various maximal cuts, allows us to focus at each stage on a small subset of contributions to an ansatz for the amplitude. In this way, we can efficiently find compact representations of amplitudes with the desired properties. The related "leading-singularity" technique [48] is also based on cutting the maximal number of propagators. In this technique, additional hidden singularities are used. This technique has been used to compute two- and three-loop planar $\mathcal{N}=4$ super-Yang-Mills amplitudes [49] with more than four external states. An interesting recent conjecture is that the leading singularities may be sufficient to determine multi-loop amplitudes in maximally supersymmetric gauge theory and gravity, and that this property may be linked to the improved ultraviolet behavior of the theories [10].

On-shell gravity and gauge theory amplitudes in four dimensions possess infrared divergences. In order to regulate these divergences, and any potential ultraviolet ones, we work in dimensional regularization (more specifically, the four-dimensional helicity scheme [50] related to dimensional reduction [51]) with $D=4-2 \epsilon$. We keep the external states in four dimensions; however, in the unitarity cuts, the cut loop momenta should be $D$ dimensional. On the other hand, the maximal-cut method is simplest to implement initially using fourdimensional instead of $D$-dimensional momenta. In principle, terms depending solely on the $(-2 \epsilon)$ dimensional components of momenta may be lost. ${ }^{1}$ To confirm that no such terms are missed, we verify that the results obtained from the maximal-cut method are valid in $D$ dimensions, using the same set of cuts used in ref. [2].

Recently Nair's on-shell super-space formalism [52] for $\mathcal{N}=4$ super-Yang-Mills MHV amplitudes in four dimensions has been extended to apply to all amplitudes [10, 26, 28, 43, 53, 54, 55]. Here we do not use these recent developments to evaluate the supermultiplet sums appearing in the cuts. Instead, when evaluating cuts in four dimensions for $\mathcal{N}=4$ super-Yang-Mills amplitudes, we make use of the observation [45] that four-point amplitudes are fully determined by considering kinematic choices that force all or nearly all intermediate

\footnotetext{
${ }^{1}$ In practice, such terms appear in theories with less than the maximal amount of supersymmetry, and in maximally supersymmetric amplitudes with more than four external states. However, they have not been found to occur for four-point amplitudes in $\mathcal{N}=4$ super-Yang-Mills theory or $\mathcal{N}=8$ supergravity, although the reason for their absence is still unclear.
} 
states to contain gluons. In this way we avoid having to perform any non-trivial sums over super-partners in the initial construction of an ansatz for the amplitude. In the course of verifying the ansatz, we need to evaluate the cuts in $D$ dimensions. Here we rely on the equivalence of $\mathcal{N}=4$ super-Yang-Mills theory to $\mathcal{N}=1$ super-Yang-Mills in ten dimensions, dimensionally reduced to four dimensions. In ten dimensions the theory consists of only a gluon and a gluino, greatly simplifying the supersymmetry bookkeeping. For the case of $\mathcal{N}=8$ supergravity, we do not need to carry out any explicit sums over the supermultiplet: the KLT relations allow us to automatically incorporate all supermultiplet sums directly from the corresponding super-Yang-Mills results.

Ref. [2] demonstrated that the three-loop four-point amplitude of $\mathcal{N}=8$ supergravity is ultraviolet finite for $D<6$. However, this left unanswered the question of whether there are any further hidden cancellations which could increase the critical dimension even further. Here we address this question by computing the divergence in $D=6$, using our new representation for the amplitude. We find that the coefficient of the (logarithmic) divergence is non-zero, implying that no further cancellations exist. This result establishes a nonvanishing coefficient for a " $D^{6} R^{4}$ " counterterm for the $D=6$ version of maximal supergravity, whose purely gravitational piece has six derivatives acting on a particular combination of four Riemann tensors. We shall also give values for the divergences in dimensional regularization in $D=7,9,11$, which are of some interest in studies of $\mathrm{M}$ theory dualities [56].

In this article, we also present the complete three-loop four-point amplitude of $\mathcal{N}=4$ super-Yang-Mills theory, in terms of a set of integrals dressed by color factors involving nonabelian structure constants. (The integrals themselves have already appeared in ref. [2].) This amplitude has strong infrared divergences in $D=4-2 \epsilon$ as $\epsilon \rightarrow 0$, which begin at order $1 / \epsilon^{6}$. The infrared properties of $\mathcal{N}=4$ super-Yang-Mills amplitudes are of some interest, both in their own right and because of their structural similarity to those of other gauge theories such as QCD. Although much of the infrared behavior of gauge theory amplitudes is well understood through the factorization and exponentiation of soft and collinear divergences [57], the color-nontrivial soft anomalous dimension matrix [58] has only been computed explicitly through two loops [59]. At this order the matrix was found to be proportional to the one-loop matrix, with a proportionality constant given by the cusp anomalous dimension [60]. It is natural to conjecture that the same property should hold at higher 
orders.

The three-loop soft anomalous dimension matrix is the only unknown quantity entering the infrared divergences of the three-loop four-point amplitude in $\mathcal{N}=4$ super-Yang-Mills theory. It does not appear in the leading-color amplitude [61]. Thus the $\epsilon$-expansion of the subleading-color terms in the complete amplitude would provide a crisp test of the proportionality of the matrix at three loops. Because soft properties are fairly insensitive to the matter content of a theory (the states with spin $<1$ ), if the proportionality holds for $\mathcal{N}=4$ super-Yang-Mills theory, it is very likely to hold for a general gauge theory. The $\epsilon$-expansion of the subleading-color terms in the amplitude requires a knowledge of the seven non-planar integrals that appear. The two planar integrals are known analytically through the finite, $\mathcal{O}\left(\epsilon^{0}\right)$, terms [61, 62]. However, the non-planar integrals pose a more difficult challenge and are as yet uncalculated.

Another application for the $\epsilon$-expansion of the non-planar integrals would be in the search for potential iterative structures in subleading-color terms, analogous to those previously found in the planar amplitudes [49, 61, 63, 64].

The infrared divergences of one-loop graviton amplitudes were studied in a classic paper by Weinberg [65]. The one-loop divergences can be exponentiated to give the leading poles in $\epsilon$ at $L$ loops, $\sim 1 / \epsilon^{L}$. The infrared behavior is less singular than in gauge theory because collinear divergences are suppressed, and simpler because no color matrices appear. However, there are still open questions about how subleading poles in $\epsilon$ behave. At two loops, there has been progress recently in showing how these poles can be iterated for the four-point $\mathcal{N}=8$ supergravity amplitude [66]. Explicit expressions for the nonplanar three-loop integrals would help check how this iteration of singular terms continues to higher order. It could also be used to search for any potential pattern of iteration for the finite terms [66].

It is well known that $\mathcal{N}=8$ supergravity contains a non-compact $E_{7(7)}$ duality symmetry [1, 67]. Its explicit action on fields in both light-cone gauge and covariant versions of the Lagrangian has been studied recently [68]. The non-trivial constraints it imposes on the emission of soft scalars at tree level have also been explored [10]. However, there is probably still more to learn about how $E_{7(7)}$ constrains multi-loop amplitudes. Some insight might be provided by the new form of the three-loop amplitude that we give here.

This paper is organized as follows. In section 1 we review the results previously obtained at two and three loops in both $\mathcal{N}=4$ super-Yang-Mills and $\mathcal{N}=8$ supergravity [2]. In 


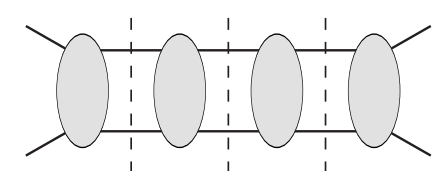

(a)

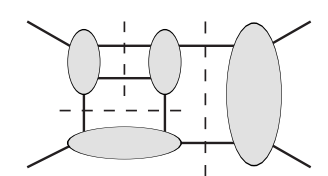

(e)

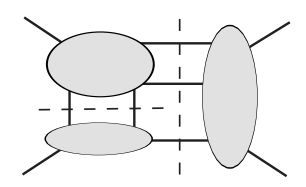

(i)

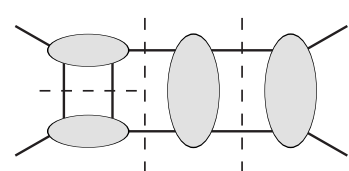

(b)

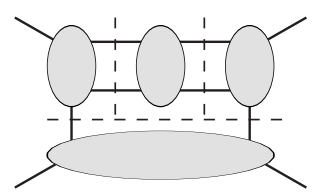

(f)

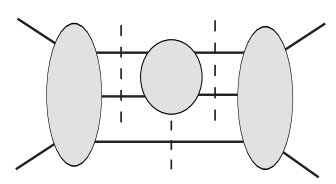

(j)

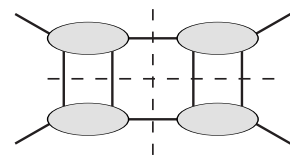

(c)

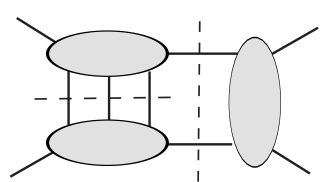

(g)

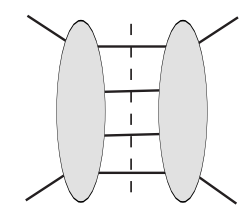

(k)

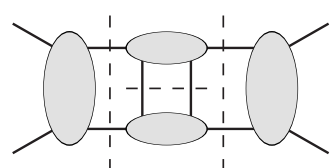

(d)

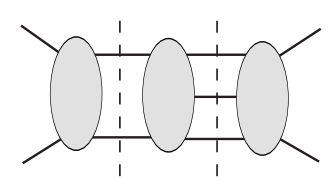

(h)

FIG. 1: A sufficient set of generalized cuts for determining any massless three-loop four-point amplitude. Each blob represents a tree amplitude. Cuts (a)-(f) are iterated two-particle cuts.

section [II we give the full color-dressed $\mathcal{N}=4$ amplitude. Then in section [V] we present a form of the $\mathcal{N}=8$ supergravity amplitude in which each term exhibits the same ultraviolet behavior as the full amplitude. In section $\nabla$ we show that the three-loop four-point amplitude diverges logarithmically in $D=6$, so there there are no further hidden cancellations. In this section we also present the three-loop divergences in $D=7,9,11$. We give our conclusions in section VI.

\section{GENERAL STRATEGY AND PREVIOUS THREE-LOOP RESULTS}

In ref. [38] the two-loop four-point $\mathcal{N}=8$ supergravity amplitude was evaluated. It was also shown that certain classes of higher-loop contributions could be evaluated to all loop orders. These so-called "iterated two-particle cut contributions" can be reduced to trees through successive two-particle cuts. The same sewing algebra appears in each two-particle cut, in essentially the same way as in $\mathcal{N}=4$ super-Yang-Mills theory [39]. In the case of this theory, examining the powers of loop momenta in the numerator of the generic iterated two-particle cut contribution suggested the finiteness bound [38],

$$
D<\frac{6}{L}+4 \quad(L>1)
$$


where $D$ is the dimension of space-time and $L$ the loop order. (The case of one loop, $L=1$, is special; the amplitudes are ultraviolet finite for $D<8$, not $D<10$.) The bound (2.1) differs somewhat from earlier superspace power counting [69], although all bounds confirm the ultraviolet (UV) finiteness of $\mathcal{N}=4$ super-Yang-Mills theory in $D=4$. This bound (2.1) has since been confirmed to all loop orders [17] using $\mathcal{N}=3$ harmonic superspace [70]. Explicit computations demonstrate that this bound is saturated through at least four loops [11, 38, 39, 63].

In ref. [38] the iterated two-particle cuts of $\mathcal{N}=8$ supergravity amplitudes were analyzed, leading to the proposal that the four-point $\mathcal{N}=8$ supergravity amplitude should be UV finite for

$$
D<\frac{10}{L}+2 \quad(L>1) .
$$

(Just as for $\mathcal{N}=4$ super-Yang-Mills theory, the one-loop case is special; $\mathcal{N}=8$ supergravity amplitudes are UV finite for $D<8$, not $D<12$ [9].) The formula (2.2) implies that in $D=4$ the first potential divergence can appear at five loops. This result was supported by studying cuts with an arbitrary number of intermediate states, but restricted to maximally-helicity-violating (MHV) amplitudes on either side of the cut. The formula is also consistent with bounds obtained by Howe and Stelle [17], assuming the existence of an $\mathcal{N}=6$ harmonic superspace [70]. However, as mentioned in the introduction, explicit three-loop computations [2] have found cancellations beyond this bound.

At three loops, the generalized cuts shown in fig. 1 are sufficient for constructing fourpoint amplitudes in any massless theory, starting from tree amplitudes. Of the cuts in fig. 1, the iterated two-particle cuts (a) to (f) were originally evaluated in refs. [38, 39] in $\mathcal{N}=4$ super-Yang-Mills and $\mathcal{N}=8$ supergravity theories. The remaining ones, (g) through (k), were evaluated in ref. [2] for both theories, determining the complete amplitudes. In general, to determine a three-loop four-point amplitude we must evaluate the cuts with distinct labels of external legs. For gravity or color-dressed gauge theory amplitudes, the various permutations of legs of the tree amplitudes composing the cuts are automatically included. However, color-ordered gauge theory tree-level partial amplitudes must be explicitly sewn in non-planar fashion to construct higher-loop non-planar subleading-color amplitudes.

The generalized-cut method for finding the amplitude is algorithmic: one first constructs an initial ansatz that reproduces one cut. Then, subsequent cuts of the amplitude are compared against the corresponding cuts of the current ansatz. If any discrepancy is found 
in a later cut, it is eliminated by adding to the ansatz terms that vanish when all the earlier cut conditions are imposed. At the end of this procedure, an integral representation of the loop amplitude is obtained with the correct cuts in all channels. This result is the complete on-shell amplitude.

In carrying out this construction, tadpole integrals and bubble contributions on external legs are dropped. Such contributions have no cuts on shell, and their integrals vanish in dimensionally-regularized amplitudes for massless particles. One might be concerned about potential UV contributions from bubble integrals on external legs, as $k_{i}^{2} \rightarrow 0$. Such terms can appear in gauge theory, and cancel collinear infrared singularities according to $1 / \epsilon_{\mathrm{UV}}+1 / \epsilon_{\mathrm{IR}}=0$. However, in $\mathcal{N}=8$ supergravity amplitudes they can be neglected, because they are suppressed by additional powers of $k_{i}^{2} \rightarrow 0$ compared to gauge theory, and also by supersymmetric cancellations.

At one loop, through the level of finite terms, massless supersymmetric amplitudes in the $\mathcal{N}=4$ theory are determined completely by their four-dimensional cuts [37]. Unfortunately, no such theorem has been demonstrated at higher loops. Because we wish to identify the smallest dimension $D$ for which an ultraviolet divergence occurs, we must ensure that all results are valid in $D$ dimensions. Evaluating the cuts in $D$ dimensions [71] makes the calculation significantly more difficult, because powerful four-dimensional spinor methods [72] can no longer be used. Some of this additional complexity is avoided by performing internal-state sums in terms of the (simpler) on-shell gauge supermultiplet of $D=10, \mathcal{N}=1$ super-YangMills theory instead of the $D=4, \mathcal{N}=4$ multiplet. By using the KLT relations [40], which are valid in any number of dimensions for the field content of maximal supergravity, the $D$-dimensional cuts of $\mathcal{N}=4$ super-Yang-Mills theory can then be reassembled into those of $\mathcal{N}=8$ supergravity [38].

In general, the KLT relations give us a rather efficient means of evaluating gravity cuts, since gauge theory amplitudes are generally simpler to evaluate than gravity amplitudes. As an example, consider the cut in fig. $\mathbb{1}(\mathrm{j})$ for $\mathcal{N}=8$ supergravity,

$$
C_{\mathcal{N}=8}^{(\mathrm{j})}=\sum_{\mathcal{N}=8 \text { states }} M_{5}^{\text {tree }}\left(1,2, l_{3}, l_{2}, l_{1}\right) \times M_{4}^{\text {tree }}\left(-l_{2},-l_{3}, l_{4}, l_{5}\right) \times M_{5}^{\text {tree }}\left(3,4,-l_{1},-l_{5},-l_{4}\right),
$$

where the sum runs over all physical states that cross any cut line. The KLT relations [40, 

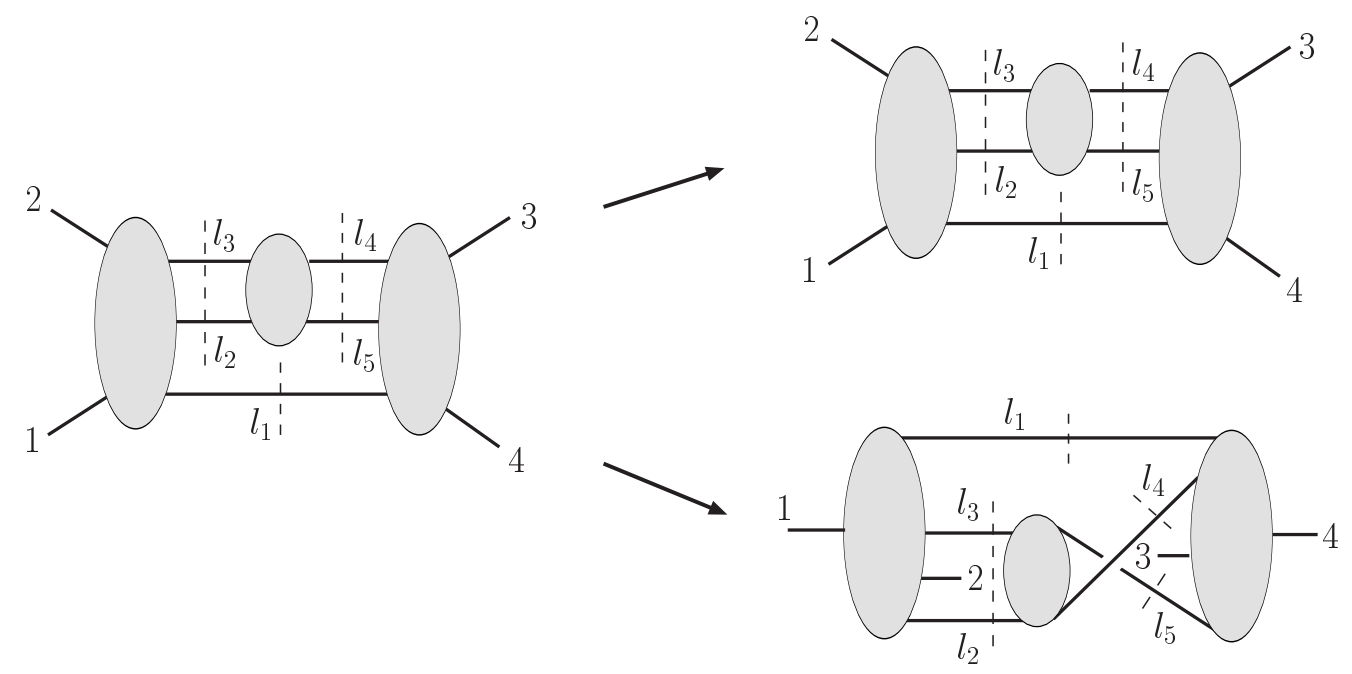

FIG. 2: The KLT relations allow us to map the gravity cuts into sums over pairs of gauge theory cuts. Here we display a pair of gauge theory cuts needed to evaluate the gravity cut shown in fig. 1(j). The remaining pairs are obtained by permuting the external legs $1 \leftrightarrow 2$ and $3 \leftrightarrow 4$.

41, 42] for these tree amplitudes are,

$$
\begin{aligned}
& M_{4}^{\text {tree }}\left(-l_{2},-l_{3},, l_{4}, l_{5}\right)=-i\left(l_{4}+l_{5}\right)^{2} A_{4}^{\text {tree }}\left(-l_{2},-l_{3}, l_{4}, l_{5}\right) A_{4}^{\text {tree }}\left(-l_{2},-l_{3}, l_{5}, l_{4}\right), \\
& \begin{aligned}
M_{5}^{\text {tree }}\left(1,2, l_{3}, l_{2}, l_{1}\right)=i\left(l_{1}+\right. & \left.k_{1}\right)^{2}\left(l_{3}+k_{2}\right)^{2} A_{5}^{\text {tree }}\left(1,2, l_{3}, l_{2}, l_{1}\right) A_{5}^{\text {tree }}\left(1, l_{1}, l_{3}, 2, l_{2}\right)+\{1 \leftrightarrow 2\}, \\
M_{5}^{\text {tree }}\left(3,4,-l_{1},-l_{5},-l_{4}\right)= & i\left(l_{4}-k_{3}\right)^{2}\left(l_{1}-k_{4}\right)^{2} \\
& \times A_{5}^{\text {tree }}\left(3,4,-l_{1},-l_{5},-l_{4}\right) A_{5}^{\text {tree }}\left(3,-l_{4},-l_{1}, 4,-l_{5}\right)+\{3 \leftrightarrow 4\},
\end{aligned}
\end{aligned}
$$

where we follow the notation of ref. [42]. The $A_{n}^{\text {tree }}$ are color-ordered gauge-theory tree amplitudes, while the $M_{n}^{\text {tree }}$ are supergravity tree amplitudes, with an overall factor of the coupling $(\kappa / 2)^{n-2}$ removed. Inserting the KLT relations into the cut (2.3) , we obtain,

$$
\begin{aligned}
C_{\mathcal{N}=8}^{(\mathrm{j})}= & i\left(l_{4}+l_{5}\right)^{2}\left(l_{1}+k_{1}\right)^{2}\left(l_{3}+k_{2}\right)^{2}\left(l_{4}-k_{3}\right)^{2}\left(l_{1}-k_{4}\right)^{2} \\
& \times \sum_{\mathcal{N}=4 \text { states }} A_{5}^{\text {tree }}\left(1,2, l_{3}, l_{2}, l_{1}\right) A_{4}^{\text {tree }}\left(-l_{2},-l_{3}, l_{4}, l_{5}\right) A_{5}^{\text {tree }}\left(3,4,-l_{1},-l_{5},-l_{4}\right) \\
& \times \sum_{\mathcal{N}=4 \text { states }} A_{5}^{\text {tree }}\left(1, l_{1}, l_{3}, 2, l_{2}\right) A_{4}^{\text {tree }}\left(-l_{2},-l_{3}, l_{5}, l_{4}\right) A_{5}^{\text {tree }}\left(3,-l_{4},-l_{1}, 4,-l_{5}\right) \\
& \quad+\{1 \leftrightarrow 2\}+\{3 \leftrightarrow 4\}+\{1 \leftrightarrow 2,3 \leftrightarrow 4\} .
\end{aligned}
$$

This equation gives the $\mathcal{N}=8$ supergravity cut directly in terms of products of two $\mathcal{N}=4$ super-Yang-Mills cuts. The relation is depicted in fig. 2, for one of the four terms in the sum 

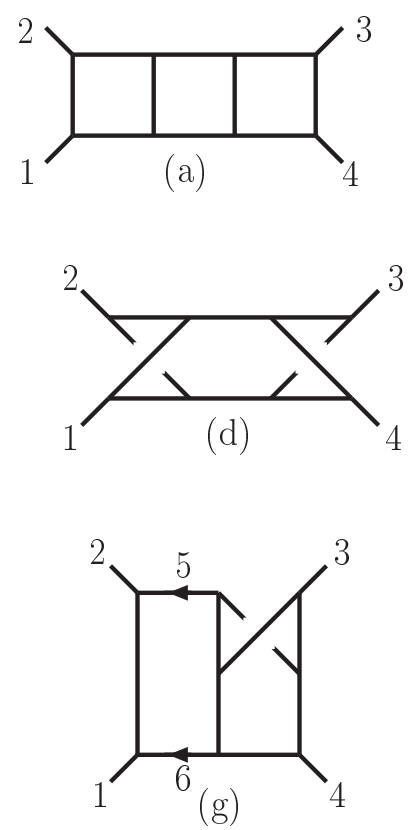
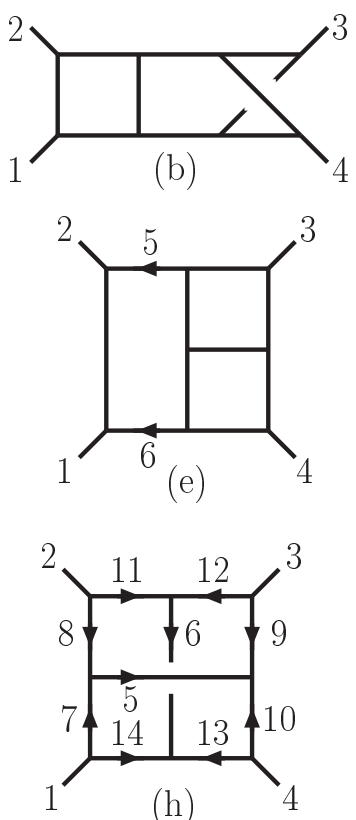
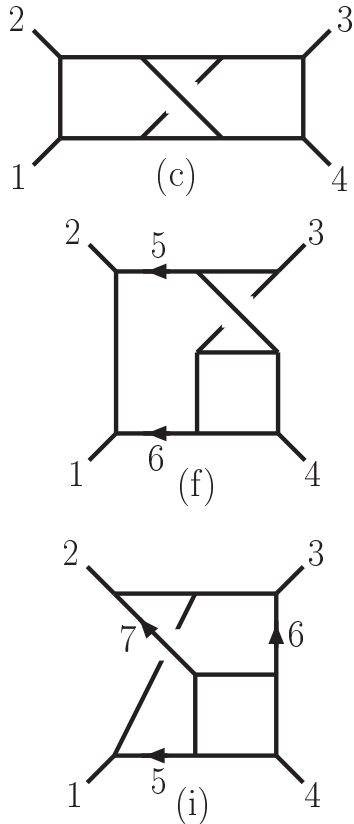

FIG. 3: The different parent integrals in terms of which four-point three-loop amplitudes may be expressed.

TABLE I: The numerator factors $N^{(x)}$ for the integrals $I^{(x)}$ in fig. 3 for $\mathcal{N}=4$ super-Yang-Mills theory. The first column labels the integral, the second column the relative numerator factor. An overall factor of $s_{12} s_{14} A_{4}^{\text {tree }}$ has been removed. The invariants $s_{i j}$ and $\tau_{i j}$ are defined in eq. (2.6).

\begin{tabular}{||c|c|}
\hline Integral $I^{(x)}$ & $N^{(x)}$ for $\mathcal{N}=4$ Super-Yang-Mills \\
\hline \hline$(\mathrm{a})-(\mathrm{d})$ & $s_{12}^{2}$ \\
\hline$(\mathrm{e})-(\mathrm{g})$ & $s_{12} s_{46}$ \\
\hline$(\mathrm{h})$ & $s_{12}\left(\tau_{26}+\tau_{36}\right)+s_{14}\left(\tau_{15}+\tau_{25}\right)+s_{12} s_{14}$ \\
\hline$(\mathrm{i})$ & $s_{12} s_{45}-s_{14} s_{46}-\frac{1}{3}\left(s_{12}-s_{14}\right) l_{7}^{2}$ \\
\hline
\end{tabular}

over external-leg permutations. One of the gauge-theory cuts is planar, while the second is nonplanar. ${ }^{2}$

An important feature of this construction is that, once the sums over all super-partners are performed in the $\mathcal{N}=4$ super-Yang-Mills cuts, the corresponding super-partner sum

\footnotetext{
${ }^{2}$ It is possible to use the total $S_{3}$ permutation symmetry of $s_{12} s_{14} A_{4}^{\text {tree }}(1,2,3,4)$ to partially "untwist" the four-point amplitude in the second Yang-Mills cut, so as to make manifest its reflection symmetry under $\{1 \leftrightarrow 4,2 \leftrightarrow 3\}$.
} 
TABLE II: The numerator factors $N^{(x)}$ for the integrals $I^{(x)}$ in fig. 3 for $\mathcal{N}=8$ supergravity, as determined in ref. [2]. The first column labels the integral, the second column the relative numerator factor. In this form of the amplitude, individual terms behave worse in the ultraviolet than does the sum over all contributions.

\begin{tabular}{||c|c||}
\hline Integral $I^{(x)}$ & $N^{(x)}$ for $\mathcal{N}=8$ Supergravity \\
\hline \hline$(\mathrm{a})-(\mathrm{d})$ & {$\left[s_{12}^{2}\right]^{2}$} \\
\hline$(\mathrm{e})-(\mathrm{g})$ & {$\left[s_{12} s_{46}\right]^{2}$} \\
\hline$(\mathrm{h})$ & $\left(s_{12} s_{89}+s_{14} s_{11,14}-s_{12} s_{14}\right)^{2}-s_{12}^{2}\left(2\left(s_{89}-s_{14}\right)+l_{6}^{2}\right) l_{6}^{2}-s_{14}^{2}\left(2\left(s_{11,14}-s_{12}\right)+l_{5}^{2}\right) l_{5}^{2}$ \\
& $-s_{12}^{2}\left(2 l_{8}^{2} l_{10}^{2}+2 l_{7}^{2} l_{9}^{2}+l_{8}^{2} l_{7}^{2}+l_{9}^{2} l_{10}^{2}\right)-s_{14}^{2}\left(2 l_{11}^{2} l_{13}^{2}+2 l_{12}^{2} l_{14}^{2}+l_{11}^{2} l_{12}^{2}+l_{13}^{2} l_{14}^{2}\right)+2 s_{12} s_{14} l_{5}^{2} l_{6}^{2}$ \\
\hline$(\mathrm{i})$ & $\left(s_{12} s_{45}-s_{14} s_{46}\right)^{2}-\left(s_{12}^{2} s_{45}+s_{14}^{2} s_{46}+\frac{1}{3} s_{12} s_{13} s_{14}\right) l_{7}^{2}$ \\
\hline
\end{tabular}

in $\mathcal{N}=8$ supergravity follows simply from the KLT relations. In fact, any simplifications performed on the gauge theory cuts can be immediately carried over to gravity cuts.

In the calculation of ref. [2], cut (i) in fig. 1 was used as the starting point. In fact, this cut detects all terms in the complete three-loop four-point amplitude for $\mathcal{N}=4$ super-Yang-Mills theory, and almost all terms for the case of $\mathcal{N}=8$ supergravity. The result of matching cut (i) with an ansatz for the $\mathcal{N}=4$ super-Yang-Mills amplitude, and then checking the result on the other cuts in fig. 1, is that the amplitude can be expressed as a linear combination of the parent integrals shown in fig. 3, with numerator factors $N^{(x)}$ given in table [1. Similarly, the three-loop four-point amplitude of $\mathcal{N}=8$ supergravity can be expressed in terms of the same parent integrals, but with different numerators. The numerators for this case, as determined in ref. [2], are shown in table II. In fig. 3 the (outgoing) momenta of the external legs are denoted by $k_{i}$ with $i=1,2,3,4$, while the momenta of the internal legs are denoted by $l_{i}$ with $i \geq 5$. For convenience we define

$$
\begin{aligned}
& s_{i j}=\left(k_{i}+k_{j}\right)^{2}, \quad \tau_{i j}=2 k_{i} \cdot k_{j}, \quad(i, j \leq 4) \\
& s_{i j}=\left(k_{i}+l_{j}\right)^{2}, \quad \tau_{i j}=2 k_{i} \cdot l_{j}, \quad(i \leq 4, j \geq 5) \\
& s_{i j}=\left(l_{i}+l_{j}\right)^{2}, \quad \tau_{i j}=2 l_{i} \cdot l_{j} . \quad(i, j \geq 5)
\end{aligned}
$$

We have altered the labeling compared to ref. [2], as a notational convenience, which will allow us to write somewhat more compactly the new representation of the $\mathcal{N}=8$ supergravity 
amplitude constructed here.

By definition, the integrals composing the amplitudes are of the form

$$
I^{(x)}=(-i)^{3} \int\left[\prod_{i=1}^{3} \frac{d^{D} q_{i}}{(2 \pi)^{D}}\right] \frac{N^{(x)}}{\prod_{j=5}^{14} l_{j}^{2}},
$$

where the $q_{i}$ 's are three independent loop momenta, the $l_{i}$ 's are the momenta of the propagators of the diagrams, and the $N^{(x)}$ are the numerator factors appearing in tables 【and II. For example, the contribution of diagram (e) of fig. 3 to the $\mathcal{N}=8$ supergravity amplitude is found by combining its propagators with the numerator $N^{(\mathrm{e})}=\left[s_{12} s_{46}\right]^{2} \equiv\left[s_{12}\left(k_{4}+l_{6}\right)^{2}\right]^{2}$ given in table $\Pi$, the result is the integral

$$
\begin{aligned}
I^{(\mathrm{e})}=(-i)^{3} \int \frac{d^{D} l_{6}}{(2 \pi)^{D}} \int \frac{d^{D} l_{7}}{(2 \pi)^{D}} \int \frac{d^{D} l_{8}}{(2 \pi)^{D}} \frac{\left[s_{12}\left(k_{4}+l_{6}\right)^{2}\right]^{2} l_{7}^{2} l_{8}^{2}\left(l_{6}-k_{1}\right)^{2}\left(l_{6}-k_{1}-k_{2}\right)^{2}\left(l_{7}-k_{4}\right)^{2}\left(l_{8}-k_{3}\right)^{2}}{} & \\
& \times \frac{1}{\left(l_{6}+l_{7}\right)^{2}\left(k_{1}+k_{2}-l_{6}+l_{8}\right)^{2}\left(k_{1}+k_{2}+l_{7}+l_{8}\right)^{2}}
\end{aligned}
$$

The complete supergravity amplitude is given in terms of the integrals in fig. 3

$$
\begin{aligned}
M_{4}^{(3)}=\left(\frac{\kappa}{2}\right)^{8} s_{12} s_{13} s_{14} M_{4}^{\text {tree }} \sum_{S_{3}} & {\left[I^{(\mathrm{a})}+I^{(\mathrm{b})}+\frac{1}{2} I^{(\mathrm{c})}+\frac{1}{4} I^{(\mathrm{d})}\right.} \\
& \left.+2 I^{(\mathrm{e})}+2 I^{(\mathrm{f})}+4 I^{(\mathrm{g})}+\frac{1}{2} I^{(\mathrm{h})}+2 I^{(\mathrm{i})}\right] .
\end{aligned}
$$

where the numerators of each integral are given in table II, $\kappa$ is the gravitational coupling, and $M_{4}^{\text {tree }}$ is the supergravity tree amplitude. In each term, $S_{3}$ denotes the set of six permutations of three external legs, say $\{2,3,4\}$, which lead from $\left(s_{12}, s_{23}\right)$ to the six independent ordered pairs of Mandelstam invariants $s_{12}, s_{13}, s_{14}$, serving as arguments of the integral. The numerical coefficients in front of each integral in eq. (2.9) are symmetry factors. They equal $4 / S$, where $S$ is the number of elements in the discrete symmetry group of the diagram. Due to supersymmetry Ward identities, the expression (2.9) is valid for any of the $256^{4}$ combinations of four particles from the 256 -dimensional $\mathcal{N}=8$ multiplet. A remarkable property of the result is that the dimension $D$ appears explicitly only in the loop integration measure; in theories with less than maximal supersymmetry we have no reason to believe that this property will continue to hold.

In this presentation of the amplitude, the UV behavior of the integrals (e)-(i) is worse than that of the full amplitude. In particular, the numerator factors for these integrals are quartic in the loop momenta. However, as discussed in ref. [2], non-trivial cancellations between diagrams cause the overall degree of divergence to be milder, in line with the behavior of 
the corresponding amplitude of $\mathcal{N}=4$ super-Yang-Mills theory. In that amplitude, table I shows that each term is quadratic in the loop momenta. In the case of a quartic behavior for $N^{(x)}$, the condition for an integral $I^{(x)}$ in eq. (2.7) to be finite in $D$ dimensions is $3 D+4<20$, or $D<16 / 3$. This inequality corresponds to the $\mathcal{N}=8$ finiteness bound (2.2) proposed in ref. [38]. In the case of a quadratic $N^{(x)}$, the finiteness condition for $I^{(x)}$ is improved to $3 D+2<20$, or $D<6$.

In ref. [2] a cancellation between integrals (e)-(i) in fig. 3 was found using table II, and working in the "vacuum approximation" in which external momenta were neglected. This approximation was adequate for demonstrating finiteness for $D<6$, but not for determining the coefficient of the $D=6$ (potential) divergence. Below, in section IV, we will present a non-trivial rearrangement of the results in table II], so that each $N^{(x)}$ is quadratic in the loop momenta, and hence each contribution satisfies the bound (2.1) with $L=3$. In section $\nabla$ we will then integrate each contribution near $D=6$, in order to compute the logarithmic divergence.

\section{COLOR DRESSING $\mathcal{N}=4$ SUPER-YANG-MILLS AMPLITUDES}

Before turning to the case of $\mathcal{N}=8$ supergravity, we first present the complete colordressed amplitude of $\mathcal{N}=4$ super-Yang-Mills theory. Although the contributing integrals were presented in ref. [2] for use as input into the supergravity calculation, they were not explicitly assembled into the complete color-dressed amplitude. Here we present the explicit color dressing in terms of Lie algebra structure constants, $f^{a b c}$. This type of dressing is natural $^{3}$ for Feynman diagrams with particles in the adjoint representation. It has been used to color-decompose tree and one-loop amplitudes [73], and to prove the Kleiss-Kuijf relations between tree-level color-ordered partial amplitudes [74]. It has also played an important role in the recent discovery of additional non-trivial tree-level identities [44].

Color dressing in terms of structure constant factors is very simple for non-contact contributions. Such terms, in which no propagator is canceled by a numerator factor, are those in the numerator factors $N^{(x)}$ in table \ that do not contain a factor of $l_{i}^{2}$. (The only explicit factor of $l_{i}^{2}$ appears in $N^{(\mathrm{i})}$; there are also implicit factors of $l_{i}^{2}$ in expressions such

\footnotetext{
${ }^{3}$ One may, alternatively, use a color dressing in terms of group generators in the fundamental representation; such dressings maintain a close relation with the double-line notation.
} 
as $\tau_{26}=s_{26}-l_{6}^{2}$ in integral (h).) The non-contact contributions are detectable from the maximal cuts, which have only three-point amplitudes. In $\mathcal{N}=4$ super-Yang-Mills theory, all such amplitudes are proportional to a factor of $f^{a b c}$. Thus the appropriate color factor for a non-contact term in a numerator $N^{(x)}$ is found simply by dressing each three-point vertex in the corresponding parent graph $(x)$ in fig. [3 with an $f^{a b c}$.

The color factors for contact-term contributions with a canceled propagator are somewhat less obvious, because they are determined from cuts containing four- or higher-point amplitudes. Such amplitudes are not proportional to a single product of $f^{a b c}$ 's, but contain multiple terms. The color factors can always be expressed as sums of products of $f^{a b c}$ 's, but it is conceivable that not all such products would be of the same form as one of the (nonvanishing) non-contact terms. However, for the three-loop four-point amplitude, we find that they are all of the same form, so that the contact terms in each $N^{(x)}$ can be consistently dressed with the same graphical color factor as the non-contact terms. We have confirmed the consistency of this assignment by evaluating all the generalized cuts in fig. 1 using tree amplitudes dressed with full color factors as the building blocks. The evaluation of these cuts is a complete check of the color dressing. The check requires use of color Jacobi identity rearrangements of the type described in ref. [73]. For general amplitudes, we expect the same property to hold: Once the contact terms are assigned to parent integrals in a way consistent with unitarity cuts that use color-ordered amplitudes, then the full color-dressed amplitudes should be obtained simply by dressing the parent integrals with an $f^{a b c}$ at each three vertex.

In summary, the fully color-dressed three-loop four-point $\mathcal{N}=4$ super-Yang-Mills amplitude is given by,

$$
\begin{aligned}
\mathcal{A}_{4}^{(3)}=-\frac{1}{4} g^{8} s_{12} s_{14} & A_{4}^{\text {tree }} \sum_{S_{4}}\left[C^{(\mathrm{a})} I^{(\mathrm{a})}+C^{(\mathrm{b})} I^{(\mathrm{b})}+\frac{1}{2} C^{(\mathrm{c})} I^{(\mathrm{c})}+\frac{1}{4} C^{(\mathrm{d})} I^{(\mathrm{d})}\right. \\
& \left.+2 C^{(\mathrm{e})} I^{(\mathrm{e})}+2 C^{(\mathrm{f})} I^{(\mathrm{f})}+4 C^{(\mathrm{g})} I^{(\mathrm{g})}+\frac{1}{2} C^{(\mathrm{h})} I^{(\mathrm{h})}+2 C^{(\mathrm{i})} I^{(\mathrm{i})}\right],
\end{aligned}
$$

where $g$ is the gauge coupling, $C^{(x)}$ are the color factors, and $I^{(x)}(s, t)$ are $D$-dimensional loop integrals corresponding to the nine diagrams in fig. 3 . The sum runs over the 24 independent permutations of legs $\{1,2,3,4\}$, denoted by $S_{4}$. The permutations in $S_{4}$ act on both kinematic and color labels. In the case of the gravity amplitude (2.9) the $S_{4}$ sum could be collapsed to an $S_{3}$ sum, holding leg 1 fixed, because the summand exhibits an additional symmetry due to the kinematic identities $s_{12}=s_{34}, s_{13}=s_{24}$, and $s_{14}=s_{23}$. In the Yang- 


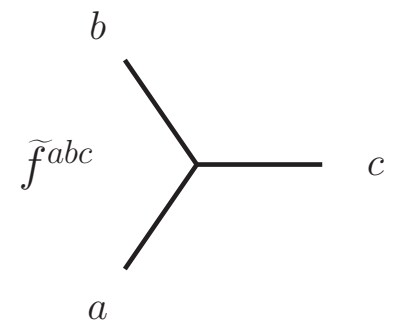

FIG. 4: Color factors for the super-Yang-Mills amplitude are obtained simply by dressing the diagrams in fig. 3 with an $\tilde{f}^{a b c}$ at each three vertex. All contact terms pick up the same color factor as the parent (non-contact) diagrams with which they are associated.

Mills case, the presence of color factors with no such manifest symmetry prevents us from collapsing the sum. Other than this minor difference, note the similarity of the gravity (2.9) and gauge theory amplitudes (3.1), including the symmetry factors of each integral. In eq. (3.1), $A_{4}^{\text {tree }}$ is the color-ordered tree amplitude $A_{4}^{\text {tree }}(1,2,3,4)$. With the factor of $s_{12} s_{14}$, it has the required overall crossing and Bose symmetry of color-dressed gauge theory amplitudes.

As illustrated in fig. 4, the color factors $C^{(x)}$ are given by dressing each three-vertex of the parent diagrams in fig. 3 with modified structure constants,

$$
\tilde{f}^{a b c}=i \sqrt{2} f^{a b c}=\operatorname{Tr}\left(\left[T^{a}, T^{b}\right] T^{c}\right),
$$

where $f^{a b c}$ are the standard structure constants, and the hermitian generators are normalized via $\operatorname{Tr}\left[T^{a} T^{b}\right]=\delta^{a b}$. The $\tilde{f}^{a b c}$ should follow the clockwise ordering of the parent diagram vertices, respecting the ordering of each vertex in fig. 3. The color factor associated with each integral is then easy to write down. For example, for diagrams (a) and (i) we have,

$$
\begin{aligned}
& C^{(\mathrm{a})}=\tilde{f}^{a_{1}, a_{5}, a_{6}} \tilde{f}^{a_{2}, a_{9}, a_{5}} \tilde{f}^{a_{3}, a_{13}, a_{14}} \tilde{f}^{a_{4}, a_{11}, a_{13}} \tilde{f}^{a_{6}, a_{7}, a_{8}} \tilde{f}^{a_{7}, a_{9}, a_{12}} \tilde{f}^{a_{8}, a_{10}, a_{11}} \tilde{f}^{a_{10}, a_{12}, a_{14}}, \\
& C^{(\mathrm{i})}=\tilde{f}^{a_{1}, a_{8}, a_{5}} \tilde{f}^{a_{2}, a_{10}, a_{7}} \tilde{f}^{a_{3}, a_{6}, a_{9}} \tilde{f}^{a_{4}, a_{13}, a_{12}} \tilde{f}^{a_{5}, a_{11}, a_{13}} \tilde{f}^{a_{6}, a_{12}, a_{14}} \tilde{f}^{a_{7}, a_{14}, a_{11}} \tilde{f}^{a_{8}, a_{10}, a_{9}} .
\end{aligned}
$$

The other factors work similarly. The factors for the different permutations in eq. (3.1) are obtained by permuting the external labels $\{1,2,3,4\}$. 

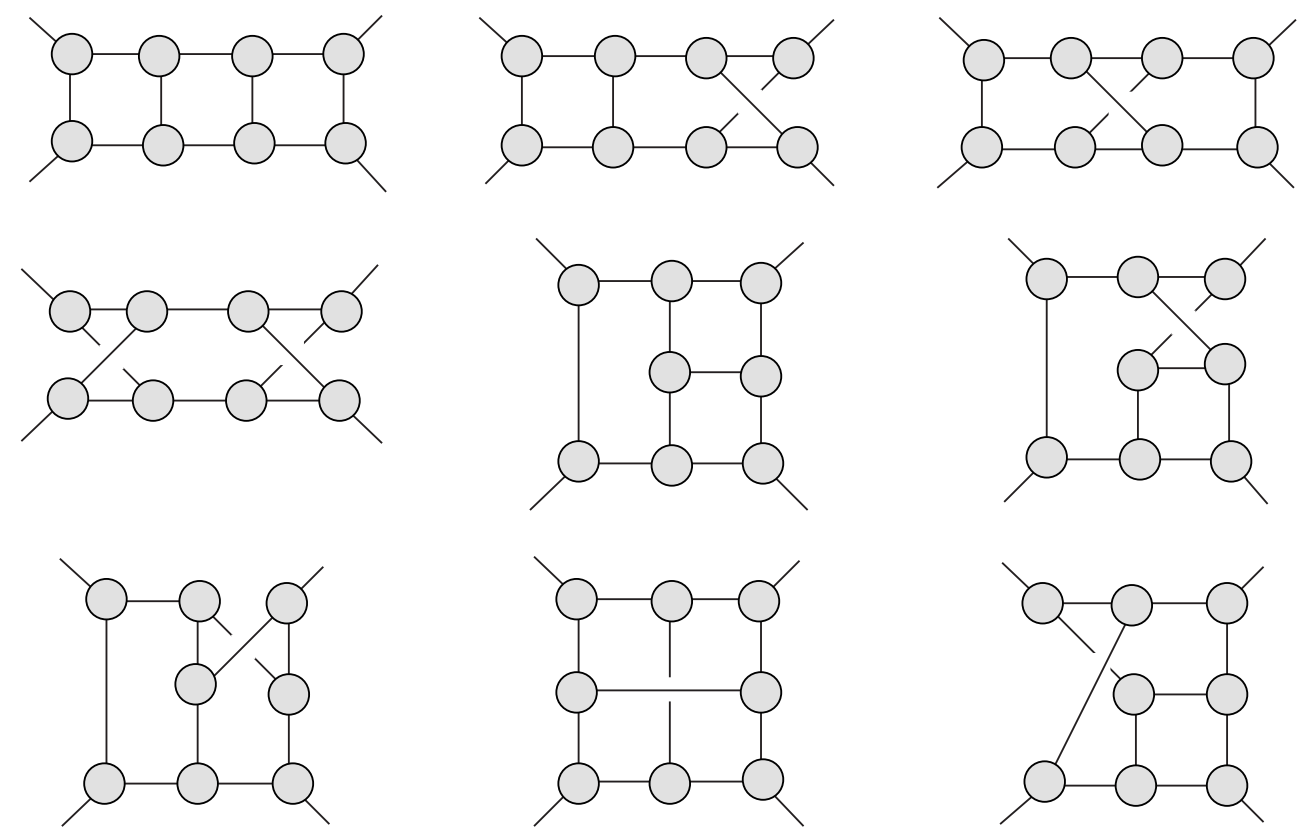

FIG. 5: The nine maximal cuts used to determine the integrands, up to contact terms. All propagator lines are taken to be cut, with the momenta satisfying on-shell conditions. The vertices represent on-shell three-point amplitudes.

\section{IV. $\mathcal{N}=8$ SUPERGRAVITY AMPLITUDE WITH MANIFEST ULTRAVIOLET BEHAVIOR}

The three-loop four-point $\mathcal{N}=8$ amplitude presented in ref. [2] has better UV behavior than each integral taken separately. It is therefore natural to try to find a representation in which each integral exhibits the behavior of the complete amplitude. One may attempt to manipulate the numerator factors in table III, by moving terms between different integral contributions until the leading two powers of loop momenta cancel. This procedure turns out to be significantly more difficult than simply reconstructing the amplitude from scratch using the method of maximal cuts [45], as we do here. By isolating in any one cut a small number of terms in the amplitude, it becomes much simpler to find ansätze for new compact forms of the amplitude, and to arrange the amplitude so that no term has a worse behavior than the complete amplitude.

We start with an ansatz for the amplitude in terms of Feynman integrals with numerator polynomials containing arbitrary parameters. We require that each numerator polynomial 
is at most quadratic in the loop momenta,

$$
N^{(x)}=\sum a_{i j}^{(x)} l_{i} \cdot l_{j}+\sum b_{i, j, m, n}^{(x)} l_{i} \cdot k_{j} l_{m} \cdot k_{n}+\sum c_{i, j}^{(x)} l_{i} \cdot k_{j}+d^{(x)},
$$

where $a_{i j}^{(x)}, b_{i, j, m, n}^{(x)}, c_{i, j}^{(x)}$ and $d^{(x)}$ are polynomials in the external momenta containing free parameters. In the sums we include only those terms not simply related to the others via momenta conservation. To determine the parameters we replace the numerators in eq. (2.9) with the numerators (4.1), and compare the cut of the ansatz against the cut of the amplitude,

$$
\sum_{\text {states }} A_{(1)}^{\text {tree }} A_{(2)}^{\text {tree }} A_{(3)}^{\text {tree }} \cdots A_{(m)}^{\text {tree }}
$$

using kinematics that place all cut lines on-shell, $l_{i}^{2}=0$. Although the comparison can be done analytically, it is generally simplest to generate kinematic solutions numerically [45]. If no solution to the cut conditions are found, then we enlarge the ansatz until one is found. For the $\mathcal{N}=8$ supergravity three-loop four-point amplitude, the quadratic numerator ansatz (4.1) is sufficient.

We start by analyzing cuts with the maximum number of cut propagators. The nine distinct - up to relabellings of external legs - maximal cuts for the three-loop four-point amplitude are displayed in fig. 5. As discussed in ref. [45], by choosing appropriate $D=4$ kinematics, we can force all cut lines to be gluons in the super-Yang-Mills case. Similarly, in the supergravity case, the same kinematics will force all cut lines to be gravitons. Remarkably, these "singlet cuts" turn out to be sufficient to determine the non-contact terms in four point amplitudes in $\mathcal{N}=4$ super-Yang-Mills theory and in $\mathcal{N}=8$ supergravity, in all known cases.

Contact terms, containing numerator factors of $l_{i}^{2}$, cannot be determined from maximal cuts, because the $l_{i}^{2}$ are all set to zero in these kinematics. To determine the contact terms, we systematically reduce the number of cut lines, until all potential contact terms are identified. In fig. 6, we show some near-maximal cuts that are particularly helpful for determining all the contact terms in the three-loop four-point amplitudes. In this figure every line represents a cut propagator (i.e., with the momenta taken on shell). In these cases, again by appropriate choices of kinematics [45], we can force almost all lines to be gluons or gravitons.

By carrying out this procedure in $D$ dimensions, we can apply the maximal cut method to any theory, including QCD. However, to take advantage of four-dimensional spinor simplifi- 

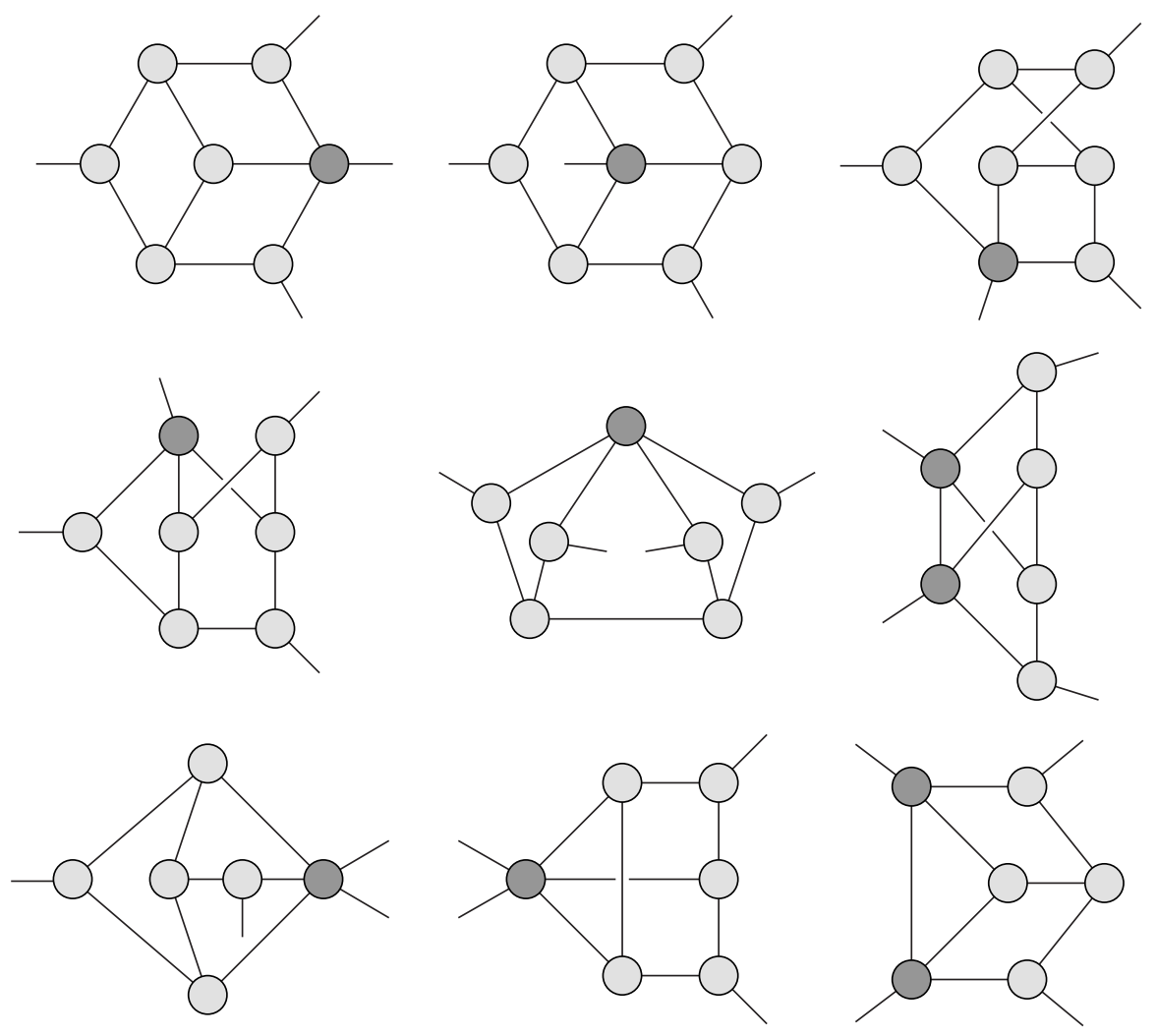

FIG. 6: Nine near-maximal cuts that are especially helpful for obtaining the contact terms for the new form of the supergravity amplitude. All propagator lines are taken to be cut, with the momenta satisfying on-shell conditions. The darker vertices represent on-shell four- and five-point amplitudes; the lighter ones depict three-point amplitudes.

cations and singlet cuts, we restrict the momenta to be four-dimensional, which potentially can drop contributions. For maximally supersymmetric four-point amplitudes, it turns out that the four-dimensional cuts appear to be sufficient for determining all contributions. (As mentioned in the introduction, in non-maximally supersymmetric theories, and in maximally supersymmetric amplitudes with more than four external states, amplitudes typically contain extra terms proportional to the $(-2 \epsilon)$ dimensional components of the loop momenta.) We can also take advantage of a variety of pictorial rules [39, 44, 45, 48] for obtaining more complicated contributions from simpler ones.

To find compact forms of the $\mathcal{N}=8$ amplitude we evaluated 98 possible maximal and near-maximal cut topologies that contain up to two contact terms. The nine maximal cuts are depicted in fig. 5. The set of maximal cuts begins with all graphs with only cubic vertices. For the applications to four-point amplitudes in maximally supersymmetric theories, we can 


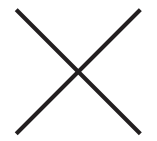

(a)

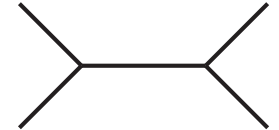

(b)

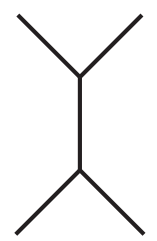

(c)

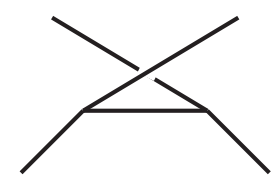

(d)

FIG. 7: A contact term can be assigned to parent diagrams by inserting a propagator multiplied by a numerator factor which cancels it. A contact term (a) can be distributed amongst the three propagator diagrams (b), (c) and (d), by including appropriate numerator factors to cancel the propagators.

discard the subset of graphs which contain triangle subgraphs or those which are one-particle reducible. We can also discard graphs containing a two-particle cut that exposes such graphs at two loops. Such cuts will lead to vanishing contributions. The surviving nine maximal cuts are in one-to-one correspondence with the nine parent integrals depicted in fig. 3. Then 27 distinct single contact term diagrams are obtained from the nine maximal cut topologies by systematically collapsing one propagator, removing ones related by symmetry. Similarly 62 double contact terms are obtained by collapsing two propagators, again removing ones related by symmetry. The 18 cuts illustrated in figs. 5 and 6 turn out to be sufficient for finding a representation of the amplitude which exhibits quadratic dependence on the loop momenta in the numerators, and thus manifestly obeys the bound (2.1) for $L=3$. Of course, these cuts by themselves do not rule out other potential contributions, including those with three or more collapsed propagators. For this we rely on the generalized cuts in fig. 1, evaluated in $D$ dimensions.

In general there is a large freedom in assigning numerator factors containing loop momenta to parent integrals, especially when no color factors are present to distinguish different integrals, as in the supergravity case. For example, scalar products of loop momenta with external momenta can often be rearranged using the observation that such products can be expressed as differences of inverse propagators, e.g., $2 k_{i} \cdot l_{j}=\left(k_{i}+l_{j}\right)^{2}-l_{j}^{2}$. As another particularly simple example, note that when assigning contact terms to parent diagrams, as illustrated in fig. 7, any four-point contact term (a) can be expressed as a combination of propagator diagrams (b)-(d) with appropriate numerator factors. The precise form is 
TABLE III: A form for the $\mathcal{N}=8$ supergravity numerator factors $N^{(x)}$ for the integrals $I^{(x)}$ in fig. 3 appearing in eq. (2.9), in which all terms are at most quadratic in the loop momenta. The first column labels the integral, the second column the relative numerator factor.

\begin{tabular}{||c|c||}
\hline Integral $I^{(x)}$ & $N^{(x)}$ for $\mathcal{N}=8$ Supergravity \\
\hline \hline$(\mathrm{a})-(\mathrm{d})$ & {$\left[s_{12}^{2}\right]^{2}$} \\
\hline$(\mathrm{e})-(\mathrm{g})$ & $s_{12}^{2} \tau_{35} \tau_{46}$ \\
\hline$(\mathrm{h})$ & $\left(s_{12}\left(\tau_{26}+\tau_{36}\right)+s_{14}\left(\tau_{15}+\tau_{25}\right)+s_{12} s_{14}\right)^{2}$ \\
& $+\left(s_{12}^{2}\left(\tau_{26}+\tau_{36}\right)-s_{14}^{2}\left(\tau_{15}+\tau_{25}\right)\right)\left(\tau_{17}+\tau_{28}+\tau_{39}+\tau_{4,10}\right)$ \\
& $+s_{12}^{2}\left(\tau_{17} \tau_{28}+\tau_{39} \tau_{4,10}\right)+s_{14}^{2}\left(\tau_{28} \tau_{39}+\tau_{17} \tau_{4,10}\right)+s_{13}^{2}\left(\tau_{17} \tau_{39}+\tau_{28} \tau_{4,10}\right)$ \\
\hline$(\mathrm{i})$ & $\left(s_{12} \tau_{45}-s_{14} \tau_{46}\right)^{2}-\tau_{27}\left(s_{12}^{2} \tau_{45}+s_{14}^{2} \tau_{46}\right)-\tau_{15}\left(s_{12}^{2} \tau_{47}+s_{13}^{2} \tau_{46}\right)$ \\
& $-\tau_{36}\left(s_{14}^{2} \tau_{47}+s_{13}^{2} \tau_{45}\right)+l_{5}^{2} s_{12}^{2} s_{14}+l_{6}^{2} s_{12} s_{14}^{2}-\frac{1}{3} l_{7}^{2} s_{12} s_{13} s_{14}$ \\
\hline
\end{tabular}

subject only to the constraint that the contributions add up to the contact term. Moreover, by using momentum conservation (and on-shell conditions on any cut legs) we can move contributions around within a larger diagram.

A particularly compact form for the supergravity amplitude is shown in table [II. The complete amplitude is given by eq. (2.9), in which the numerators $N^{(x)}$ in table 1 are replaced by those in table III. Each numerator respects the symmetry of its corresponding diagram. Because the new form matches the $D$-dimensional cuts displayed in fig. 1, it is completely equivalent to the earlier form. The two forms are, however, rather non-trivially related. In particular, the form in table II has numerator terms which are quartic in loop momenta, while the form in fig. III is merely quadratic. The form in table III is by no means unique. The large freedom, mentioned previously, in assigning numerator factors to a parent graph implies that there are continuous families of numerators with the same quadratic behavior that satisfy all cut conditions. As a check, we have evaluated the logarithmic divergence of the various forms at $D=6$, and we find that it is independent of each of the free parameters, as expected. 


\section{DIVERGENCES IN HIGHER DIMENSIONS}

As demonstrated in ref. [38], the two-loop four-graviton $\mathcal{N}=8$ supergravity amplitude saturates the finiteness bound (2.1), having a critical dimension $D_{c}=7$. The values of the two-loop divergence in dimensions between $D=7$ and $D=11$ were also calculated in that reference, in dimensional regularization. In this section we carry out a similar analysis at three loops, using the new representations of the amplitudes given in table III. We prove that in $\mathcal{N}=8$ supergravity the bound (2.1) is saturated at $L=3$; that is, a logarithmic UV divergence is present in $D=6$. We also give the explicit values of the dimensionallyregulated power-law divergences in $D=7,9,11$. Other than the critical dimension $D_{c}=6$, odd dimensions are also interesting because of the connection to M theory dualities [56] in dimensions $D=9,11$. From a technical standpoint, it is easier to compute the three-loop divergences in odd dimensions than it is in even dimensions. In odd dimensions the only divergences that arise in dimensional regularization are from two-loop subdivergences.

\section{A. Divergence in the critical dimension $D=6$.}

We already know that for $\mathcal{N}=4$ super-Yang-Mills theory the finiteness bound is saturated through at least four loops [11]. Is the bound (2.1) saturated for $\mathcal{N}=8$ supergravity as well? It would of course be rather surprising if $\mathcal{N}=8$ supergravity were better behaved in the UV than $\mathcal{N}=4$ super-Yang-Mills theory - but surprises have happened before. It is nevertheless important to confirm that no further "hidden" cancellations exist. Because we now have a compact analytic expression for the amplitude, without additional spurious divergences that cancel between integrals, we can settle the issue simply by evaluating the integrals near $D=6$ and assembling the amplitude in this dimension. If the amplitude diverges in $D=6$, then no further cancellations exist at three loops, beyond those found in ref. [2], and exhibited manifestly by the numerator factors in table III]

In its critical dimension, where an integral first develops a divergence, the divergence is logarithmic. This implies that the residue of the $1 / \epsilon$ pole is constant, after all factors of external momentum in the numerator are taken out of the integral. To evaluate this constant we may choose the external momenta and invariants in any convenient way. In particular, we may expand the integrand for small external momenta and keep the leading 


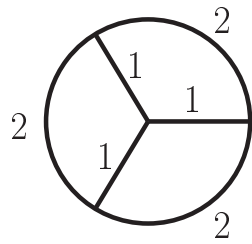

(A)

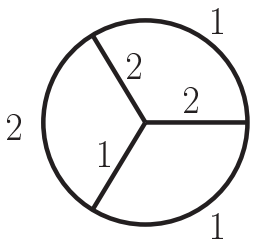

(B)

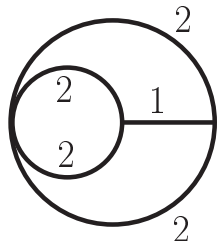

(C)

FIG. 8: Vacuum-like diagrams describing the leading behavior. The labels 1,2 indicate whether the corresponding propagator appears to the first or second power.

term. A key advantage of the new representation of the amplitude in table III, compared to the original one in table II is that no integral has a critical dimension below the expected critical dimension of the amplitude. This simplifies the evaluation of the divergence because it receives contributions only from the leading term in the small-momentum expansion.

In addition we can drop all integrals with a critical dimension larger than six. Inspecting table III, fig. (3), and eq. (2.7), we see that integrals (a)-(d) are all finite in $D=6$, so we need only evaluate the integrals (e)-(i). These integrals have numerators quadratic in the loop momenta. We expand at small external momenta, keeping only the UV-divergent integrals which are independent of the external momenta, apart from the explicit factors of external momenta appearing in the numerator. Lorentz invariance implies that we can replace

$$
\int d^{D} q_{1} d^{D} q_{2} d^{D} q_{3} q_{i}^{\mu} q_{j}^{\nu} f\left(q_{1}, q_{2}, q_{3}\right) \rightarrow \frac{\eta^{\mu \nu}}{D} \int d^{D} q_{1} d^{D} q_{2} d^{D} q_{3} q_{i} \cdot q_{j} f\left(q_{1}, q_{2}, q_{3}\right),
$$

because $\eta_{\mu \nu}$ is the only available tensor that can have a divergent coefficient. Contracting both sides with $\eta_{\mu \nu}$ shows that the prefactor on the right-hand side is indeed $1 / D$. For the purpose of computing the leading UV singularity in the critical dimension, this identity allows us to replace any two-tensor integral with simpler scalar integrals, obtained by rewriting $q_{i} \cdot q_{j}$ as a linear combination of inverse propagators.

Keeping only the leading terms at small external momenta and using eq. (5.1), we replace the diagrams in fig. 3 with the simpler vacuum diagrams shown in fig. 8 , We have,

$$
\begin{aligned}
I^{(\mathrm{a})} & \rightarrow 0, \quad I^{(\mathrm{b})} \rightarrow 0, \quad I^{(\mathrm{c})} \rightarrow 0, \quad I^{(\mathrm{d})} \rightarrow 0, \\
I^{(\mathrm{e})} & \rightarrow \frac{-s_{12}^{3}}{3} V^{(\mathrm{A})}, \quad I^{(\mathrm{f})} \rightarrow \frac{-s_{12}^{3}}{3} V^{(\mathrm{B})}, \quad I^{(\mathrm{g})} \rightarrow \frac{-s_{12}^{3}}{3} V^{(\mathrm{B})}, \\
I^{(\mathrm{h})} & \rightarrow \frac{2}{3}\left(2 s_{13}^{3}-3 s_{12} s_{13} s_{14}\right) V^{(\mathrm{B})}+\left(-s_{13}^{3}+s_{12} s_{13} s_{14}\right) V^{(\mathrm{C})}, \\
I^{(\mathrm{i})} & \rightarrow \frac{1}{6}\left(2 s_{13}^{3}-5 s_{12} s_{13} s_{14}\right) V^{(\mathrm{A})}-\frac{1}{3}\left(s_{13}^{3}+3 s_{12} s_{13} s_{14}\right) V^{(\mathrm{B})},
\end{aligned}
$$




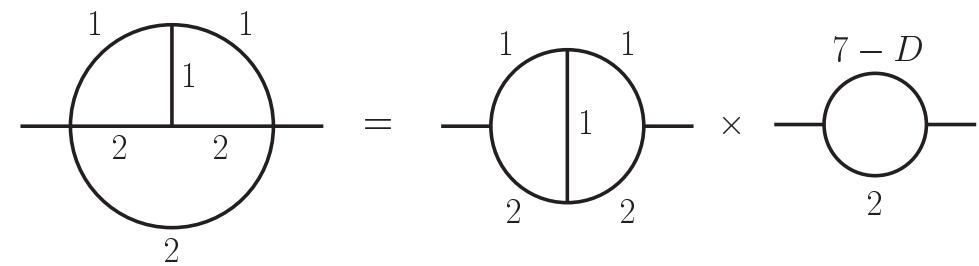

(B)

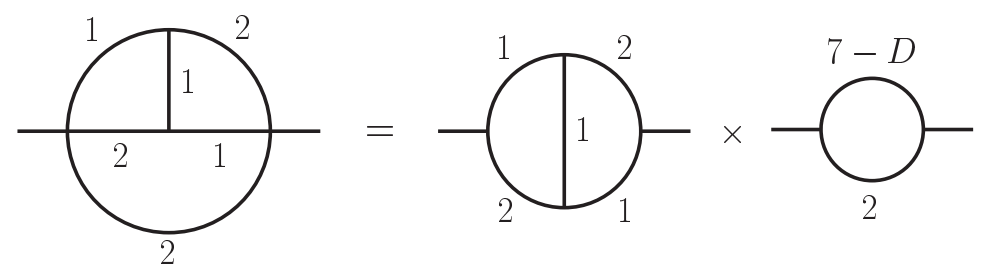

FIG. 9: Reduction of three-loop propagator diagrams appearing in the $D=6-2 \epsilon$ UV divergences of $\mathcal{N}=8$ supergravity. The numbers near each propagator indicates the power or index to which it is raised.

where we used

$$
s_{12}^{3}+s_{13}^{3}+s_{14}^{3}=3 s_{12} s_{13} s_{14}
$$

to simplify the expressions, and we set $D=6$ in the coefficients. Multiplying the above expressions by their numerical prefactors in eq. (2.9) and summing over the permutations, it is not hard to find that the leading UV divergence in $D=6$ is

$$
\left.M_{4}^{(3), D=6-2 \epsilon}\right|_{\text {pole }}=-\left(\frac{\kappa}{2}\right)^{8}\left(s_{12} s_{13} s_{14}\right)^{2} M_{4}^{\text {tree }}\left[10\left(V^{(\mathrm{A})}+3 V^{(\mathrm{B})}\right)\right]
$$

The coefficient of the vacuum-like diagram, $V^{(\mathrm{C})}$, which arises only from $I^{(\mathrm{h})}$, vanishes after the permutation sum. These features are a consequence of the specific representation of the numerators given in table III.

To determine whether the leading $1 / \epsilon$ pole in eq. (2.9) cancels, we must evaluate the UV behavior of the vacuum integrals (A) and (B) in fig. 8, A convenient means for doing so is to restore some momentum dependence in order to regulate the infrared divergences. We can re-interpret the calculation as that of a typical propagator integral, simply by injecting some arbitrary external momentum at appropriately-chosen vertices, and applying standard techniques developed for this problem. (See for example the review by Grozin [75].) Because the divergence is logarithmic in the critical dimension, the injected momentum does not appear in the leading UV pole in dimensional regularization; thus it has no effect on the result. We therefore promote the vacuum-like diagrams (A) and (B) in fig. 8 to propagator 
diagrams, while leaving all internal lines massless, as shown in fig. 9. Then we can use dimensional analysis to simplify each three-loop propagator diagram down to a product of a two-loop propagator diagram and a one-loop bubble diagram. (Although we do not need it here, diagram $(\mathrm{C})$ in fig. 8 can be evaluated in a similar fashion.)

As shown in fig. 9, to evaluate the integrals, we factor out a two-loop propagator diagram formed from the two upper loops on the left side of diagrams (A) and (B). By dimensional analysis, the two-loop propagator subintegrals are given by

$$
P_{2 \text {-loop }}^{(\mathrm{A})}=\frac{K_{2-\text { loop }}^{(\mathrm{A})}}{(4 \pi)^{D}} \frac{1}{\left(l^{2}\right)^{7-D}}, \quad \quad P_{2 \text {-loop }}^{(\mathrm{B})}=\frac{K_{2 \text {-loop }}^{(\mathrm{B})}}{(4 \pi)^{D}} \frac{1}{\left(l^{2}\right)^{7-D}},
$$

where $l$ is the momentum flowing through the two-loop propagator diagram and $K_{2-\text { loop }}^{(\mathrm{A})}$ and $K_{2 \text {-loop }}^{(\mathrm{B})}$ are constants to be determined by explicit integration. The factor of $1 /\left(l^{2}\right)^{7-D}$ effectively results in a remaining one-loop bubble integral with a propagator raised to the power $(7-D)$. The two-loop propagator integrals in each case are finite in $D=6$. We evaluated them using integration by parts [76], with the result,

$$
K_{2-\text { loop }}^{(\mathrm{A})}=1, \quad K_{2-\text { loop }}^{(\mathrm{B})}=\zeta_{3}-\frac{1}{3} .
$$

The remaining one-loop bubble integrals are also straightforward to evaluate; for arbitrary exponents $n_{1}$ and $n_{2}$ of the two propagators, they are given by [75]

$$
I^{\text {bubble }}\left(n_{1}, n_{2}\right) \equiv-i \int \frac{d^{D} p}{(2 \pi)^{D}} \frac{1}{\left((p+k)^{2}\right)^{n_{1}}\left(p^{2}\right)^{n_{2}}}=\frac{(-1)^{n_{1}+n_{2}}}{(4 \pi)^{D / 2}} G\left(n_{1}, n_{2}\right)\left(-k^{2}\right)^{-\left(n_{1}+n_{2}-D / 2\right)},
$$

where

$$
G\left(n_{1}, n_{2}\right)=\frac{\Gamma\left(-D / 2+n_{1}+n_{2}\right) \Gamma\left(D / 2-n_{1}\right) \Gamma\left(D / 2-n_{2}\right)}{\Gamma\left(n_{1}\right) \Gamma\left(n_{2}\right) \Gamma\left(D-n_{1}-n_{2}\right)} .
$$

In $D=6-2 \epsilon$, for the two cases in fig. 9 we have $n_{1}=1+2 \epsilon$ and $n_{2}=2$. The bubble integral in both cases provides the UV divergence in $D=6-2 \epsilon$,

$$
G(1+2 \epsilon, 2)=\frac{\Gamma(3 \epsilon) \Gamma(2-3 \epsilon) \Gamma(1-\epsilon)}{\Gamma(1+2 \epsilon) \Gamma(2) \Gamma(3-4 \epsilon)}=\frac{1}{6 \epsilon}+\mathcal{O}(1) .
$$

Recalling the normalization of the integrals $I^{(x)}$ defined in eq. (2.7), and collecting factors from eqs. (5.6) and (5.7), we obtain the UV singularity of the vacuum-like diagrams (A) and (B),

$$
\begin{aligned}
V^{(\mathrm{A})} & =-\frac{1}{(4 \pi)^{9}}\left[\frac{1}{6 \epsilon}+\mathcal{O}(1)\right], \\
V^{(\mathrm{B})} & =-\frac{1}{(4 \pi)^{9}}\left[\frac{1}{6 \epsilon}\left(\zeta_{3}-\frac{1}{3}\right)+\mathcal{O}(1)\right] .
\end{aligned}
$$


We have confirmed these results by direct numerical integration of the vacuum-like diagrams, using a mass regulator to define the integrals. Using eq. (5.4), we obtain

$$
\left.M_{4}^{(3), D=6-2 \epsilon}\right|_{\text {pole }}=\frac{1}{\epsilon} \frac{5 \zeta_{3}}{(4 \pi)^{9}}\left(\frac{\kappa}{2}\right)^{8}\left(s_{12} s_{13} s_{14}\right)^{2} M_{4}^{\text {tree }} \text {. }
$$

Note that the simple functional dependence of eq. (5.12) on the kinematic variables $s_{12}, s_{13}$ and $s_{14}$ is fixed by dimensional analysis and Bose symmetry, given that it should contain a factor of $s_{12} s_{13} s_{14} M_{4}^{\text {tree }}$. The identity (5.3) can be used to help establish this fact.

Notice also the cancellation of the rational parts of $K_{2-\text { loop }}^{(\mathrm{A})}$ and $K_{2-\text { loop }}^{(\mathrm{B})}$, in the combination $V^{(\mathrm{A})}+3 V^{(\mathrm{B})}$ appearing in eq. (15.4). Thanks to this cancellation, the logarithmic UV divergence (5.12) possesses a uniform degree of transcendentality (in which $\zeta_{n}$ is assigned degree $n$, and rational numbers degree zero). This property is common to infrared-regulated amplitudes, near $D=4$, in $\mathcal{N}=4$ super-Yang-Mills theory and $\mathcal{N}=8$ supergravity; here we see it persists to the level of the three-loop UV singularity at $D=6$.

As mentioned earlier, the fact that eq. (5.12) is nonzero establishes that, in comparison with $\mathcal{N}=4$ super-Yang-Mills theory, $\mathcal{N}=8$ supergravity is no better behaved (as well as no worse behaved) in the ultraviolet through three loops.

\section{B. UV divergences in odd dimensions}

Next we turn to the computation of three-loop divergences in dimensions above six. To simplify the analysis, we restrict our attention to the behavior near odd values of $D$, namely $D=7,9,11$.

According to the convergence theorem, a Feynman integral is convergent if the degree of divergence of all one-particle-irreducible subintegrals is negative. Together with the BPHZ subtraction it also implies that, after subtracting all subdivergences, the remaining overall divergence (arising when all loop momenta are scaled to infinity at the same rate) has a local (polynomial) dependence on external momentum invariants. In dimensional regularization it is easy to see that, in odd dimensions and at an odd loop order, no such overall divergences may exist. Indeed, the dimension of any such loop integral is odd, and so the result of the integration depends on a half-integer power of momentum invariants. However, such a dependence would be nonlocal. Hence its coefficient must be finite after the subtraction of all subdivergences. Thus, after subtracting the subdivergences, all three-loop integrals must 


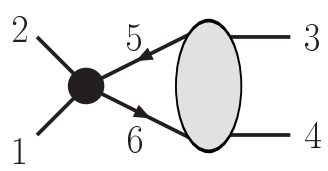

(a)

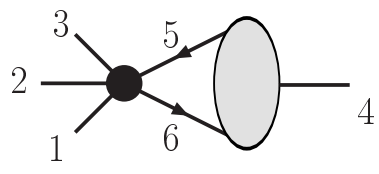

(b)

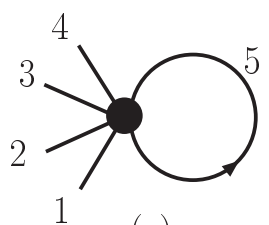

(c)

FIG. 10: The two-loop subdivergence contributions to the three-loop local divergence. The black vertex represents a two-loop local divergence. In dimensional regularization, near a dimension greater than four, contributions (b) and (c) vanish because they are proportional to a positive power of a vanishing invariant. The complete contribution is given by summing over the inequivalent permutations of external legs.

be finite in odd dimensions. For the same reason, no subtraction is necessary for one-loop subintegrals in odd dimensions. Therefore, the first divergent subintegrals have two loops, and they completely determine the leading UV divergence of the three-loop integrals to which they belong.

The above arguments hold for general off-shell integrals. Our case is somewhat simpler due to the masslessness of the external momenta. Consider the potential two-loop subdivergences shown in fig. 10, In principle, there can be contributions from two-loop four-, fiveand six-point subdivergences. (There is no three-point subdivergence in any supergravity theory.) However, from dimensional analysis in $D>4$ dimensions we can easily show that both diagrams (b) and (c) vanish, because they are proportional to vanishing invariants. For example, inserting a five-point two-loop divergence into the third loop, as shown in figure 10(b), amounts to computing

$$
\int \frac{d^{D} q}{(2 \pi)^{D}} \frac{P\left(q, k_{1}, k_{2}\right)}{q^{2}\left(q+k_{4}\right)^{2}} \sim\left(k_{4}^{2}\right)^{D-4+n_{q} / 2} .
$$

Here $P\left(q, k_{1}, k_{2}\right)$ is a polynomial depending on the loop and external momenta, and $n_{q}$ counts the power of $q$ in the numerator of a given term. Even for terms with no powers of $q$ in the numerator, for $D \geq 6$ the integral is proportional to a positive power of $k_{4}^{2}=0$ and therefore vanishes. With additional powers of loop momenta $q$ in the numerator, the vanishing is even stronger.

Similarly, integral (c) in fig. 10 must vanish, because no external invariant appears. It is interesting to note that the three-loop integrals (d), (h) and (i) in fig. 3 do not have two-loop four-point subintegrals. (This property is tied to the fact that integrals $(\mathrm{h})$ and (i) cannot 
be detected via two-particle cuts. Integral (d) can be detected in this way, but only by a cut that splits it into a product of two one-loop integrals.) Thus, only the integrals (a), (b), (c), (e), (f) and (g) in fig. 3 contribute to the divergence in odd dimensions. The first odd dimension in which a divergence appears in the three-loop amplitude is $D=7$, because this is the dimension where the first two-loop subdivergence appears [38].

To evaluate the contribution of the two-loop subdivergence, in principle we need the divergent parts of the two-loop planar and nonplanar double-box integrals with two offshell external legs. However, a simple observation allows us to obtain the desired result using only double-box integrals with all massless legs. Indeed, at the level of the singular terms in $D \geq 7$, the difference between the massive (off-shell) and massless integrals are terms proportional to the square of the massive momenta. When these additional terms are inserted in the triangle graph shown in fig. 10, they cancel at least one of the propagators of the triangle integral, leaving behind a bubble integral with a massless momentum flowing through it. As explained above, such integrals vanish in dimensional regularization.

Using this observation, an efficient strategy is to combine the divergences of the subintegrals into divergences of on-shell subamplitudes. This is done simply by grouping together the remaining one-loop integrals with the same remaining propagators, which is effectively done in fig. 10. With this reorganization we directly evaluate the divergence in fig. 10(a), with the two internal legs, 5 and 6 , placed on shell. All other contributions vanish. The contact term vertex represents the divergence of the on-shell two-loop four-point amplitude. These divergences have been computed in ref. [38] and are given by,

$$
\begin{aligned}
\left.\mathcal{M}_{4}^{2 \text {-loop, } D=7-2 \epsilon}\right|_{\text {pole }}= & \frac{1}{2 \epsilon(4 \pi)^{7}} \frac{\pi}{3}\left(s_{12}^{2}+s_{13}^{2}+s_{14}^{2}\right) \times\left(\frac{\kappa}{2}\right)^{6} \times s_{12} s_{13} s_{14} M_{4}^{\text {tree }}, \\
\left.\mathcal{M}_{4}^{2 \text {-loop, } D=9-2 \epsilon}\right|_{\text {pole }}= & \frac{1}{4 \epsilon(4 \pi)^{9}} \frac{-13 \pi}{9072}\left(s_{12}^{2}+s_{13}^{2}+s_{14}^{2}\right)^{2} \times\left(\frac{\kappa}{2}\right)^{6} \times s_{12} s_{13} s_{14} M_{4}^{\text {tree }}, \\
\left.\mathcal{M}_{4}^{2 \text {-loop, } D=11-2 \epsilon}\right|_{\text {pole }}= & \frac{1}{48 \epsilon(4 \pi)^{11}} \frac{\pi}{5791500}\left(438\left(s_{12}^{6}+s_{13}^{6}+s_{14}^{6}\right)-53 s_{12}^{2} s_{13}^{2} s_{14}^{2}\right) \\
& \times\left(\frac{\kappa}{2}\right)^{6} \times s_{12} s_{13} s_{14} M_{4}^{\text {tree }},
\end{aligned}
$$

for external legs labeled $1,2,3,4$. The factor $s_{12} s_{13} s_{14}$ cancels all kinematic poles in the tree amplitudes $M_{4}^{\text {tree }}$, making these divergences local. (In fact, the product $s_{12} s_{13} s_{14} M_{4}^{\text {tree }}$ in the case of four gravitons may be represented as the matrix element on four-particle states of a particular contraction of four Riemann tensors, often denoted simply by " $R^{4}$ ".) We have also obtained the same result (5.14) by evaluating the two-loop subdivergences integral by 
integral, using the results of ref. [38].

To evaluate the remaining one-loop integrals, we again make use of the observation that if a numerator factor collapses either of the propagators in fig. 10(a), labeled by 5 and 6 , then the integral vanishes because it depends only on a massless external momentum, either $k_{3}^{2}=0$ or $k_{4}^{2}=0$, as in fig. 10(b). Therefore we may set $l_{5}^{2}=0$ and $l_{6}^{2}=0$ in the numerators of the integrals. We may use the on-shell two-loop divergence (5.14) and tree amplitude directly in the integration, giving us

$$
\begin{aligned}
\left.\mathcal{M}_{4}^{3-\text { loop }, D}\right|_{\text {pole }}=\frac{1}{2} \sum_{\text {perms }} \sum_{\mathcal{N}=8 \text { states }} \int \frac{d^{D} l_{5}}{(2 \pi)^{D}} \\
\quad \times\left[\left.\mathcal{M}_{4}^{2-\text { loop }, D}\left(-l_{5}, 1,2, l_{6}\right)\right|_{\text {pole }}\right] \frac{i}{l_{6}^{2}} M_{4}^{\text {tree }}\left(-l_{6}, 3,4, l_{5}\right) \frac{i}{l_{5}^{2}},
\end{aligned}
$$

where the factor of $1 / 2$ accounts for identical particles crossing the cut, and the sum over permutations runs over the six distinct labellings of fig. 10(a). To evaluate this integral we note that the two-loop divergences (5.14) are proportional to the tree-level four-graviton amplitude. Thus, we may use the two-particle cut sewing relation for tree-level amplitudes [38],

$$
\begin{aligned}
& \sum_{\mathcal{N}=8 \text { states }} M_{4}^{\text {tree }}\left(-l_{5}, 1,2, l_{6}\right) \times M_{4}^{\text {tree }}\left(-l_{6}, 3,4, l_{5}\right) \\
& \quad=i s_{12} s_{13} s_{14} M_{4}^{\text {tree }}(1,2,3,4)\left[\frac{1}{\left(l_{5}-k_{1}\right)^{2}}+\frac{1}{\left(l_{5}-k_{2}\right)^{2}}\right]\left[\frac{1}{\left(l_{6}-k_{3}\right)^{2}}+\frac{1}{\left(l_{6}-k_{4}\right)^{2}}\right] .
\end{aligned}
$$

In the sewing (5.15), the additional factor $s_{12} s_{13} s_{14}$ appearing in the two-loop divergences (5.14) should be relabeled as,

$$
s_{12} s_{13} s_{14} \mapsto s_{12}\left(l_{5}-k_{1}\right)^{2}\left(l_{5}-k_{2}\right)^{2} .
$$

With this additional factor the first term in the bracket in eq. (5.16) simplifies as,

$$
\begin{aligned}
s_{12}\left(l_{5}-k_{1}\right)^{2}\left(l_{5}-k_{2}\right)^{2}\left[\frac{1}{\left(l_{5}-k_{1}\right)^{2}}+\frac{1}{\left(l_{5}-k_{2}\right)^{2}}\right] & =s_{12}\left[\left(l_{5}-k_{2}\right)^{2}+\left(l_{5}-k_{1}\right)^{2}\right] \\
& =-s_{12}^{2},
\end{aligned}
$$

where we used the on-shell conditions on $l_{5}$ and $l_{6}$. Because a propagator cancels, we are left with the expected triangle integrals in eq. (5.15).

The masslessness of the external legs leads to further simplifications in handling tensor triangle integrals, which arise from additional factors of $s_{13}$ and $s_{14}$ in the twoloop divergences (5.14). If in these factors we let $s_{13} \mapsto\left(l_{5}-k_{1}\right)^{2}=-2 l_{5} \cdot k_{1}$ and 
$s_{14} \mapsto\left(l_{6}+k_{1}\right)^{2}=2 l_{6} \cdot k_{1}$, then all contractions $l_{i}^{\mu} l_{j}^{\nu} \rightarrow \eta^{\mu \nu}$ vanish, because they are proportional to $k_{1}^{2}=0$. Hence we just need the Feynman parameter polynomials obtained by shifting the loop momentum in the usual way. Evaluating the integrals over the Feynman parameters in the appropriate odd integer dimension, and combining the pieces, we obtain the following three-loop divergences:

$$
\begin{aligned}
\left.M_{4}^{(3)}\right|_{\text {pole }} ^{D=7-2 \epsilon}= & -\frac{\pi^{5 / 2}}{\epsilon(4 \pi)^{21 / 2}}\left(\frac{\kappa}{2}\right)^{8}\left[s_{12} s_{13} s_{14} M_{4}^{\text {tree }}\right] \sum_{Z_{3}} \frac{\left(-s_{12}\right)^{5 / 2}}{1440}\left(65 s_{12}^{2}-8 s_{13} s_{14}\right) \\
\left.M_{4}^{(3)}\right|_{\text {pole }} ^{D=9-2 \epsilon}= & -\frac{\pi^{5 / 2}}{\epsilon(4 \pi)^{27 / 2}}\left(\frac{\kappa}{2}\right)^{8}\left[s_{12} s_{13} s_{14} M_{4}^{\text {tree }}\right] \sum_{Z_{3}} \frac{13\left(-s_{12}\right)^{7 / 2}}{11705057280} \\
& \times\left(10143 s_{12}^{4}-1296 s_{12}^{2} s_{13} s_{14}+128 s_{13}^{2} s_{14}^{2}\right) \\
\left.M_{4}^{(3)}\right|_{\text {pole }} ^{D=11-2 \epsilon}= & -\frac{\pi^{5 / 2}}{\epsilon(4 \pi)^{33 / 2}}\left(\frac{\kappa}{2}\right)^{8}\left[s_{12} s_{13} s_{14} M_{4}^{\text {tree }}\right] \sum_{Z_{3}} \frac{\left(-s_{12}\right)^{9 / 2}}{2461954796421120000} \\
& \times\left(3180433113 s_{12}^{6}-247667992 s_{12}^{4} s_{13} s_{14}\right. \\
& \left.+70002816 s_{12}^{2} s_{13}^{2} s_{14}^{2}-3363840 s_{13}^{3} s_{14}^{3}\right)
\end{aligned}
$$

where $Z_{3}$ refers to cyclic permutations of legs 2,3 and 4 . These divergences are really due to two-loop subdivergences in the bare theory. If we renormalize the theory at two loops in $D=7,9$ or 11 , in order to cancel the divergences in eq. (5.14), then the corresponding three-loop divergence in the renormalized theory will also be cancelled.

\section{CONCLUSIONS}

Maximally supersymmetric $\mathcal{N}=8$ supergravity theory is potentially a perturbatively ultraviolet-finite point-like quantum field theory of gravity. In ref. [2] a loop-integral representation of the three-loop four-point amplitude of $\mathcal{N}=8$ supergravity was presented, which exhibited cancellations beyond those needed for finiteness. In this paper, using the method of maximal cuts [45], we constructed an alternate representation of this amplitude with all ultraviolet cancellations manifest. By explicitly evaluating the integrals, we demonstrated that $\mathcal{N}=8$ supergravity [1] diverges in $D=6$, matching the ultraviolet behavior of $\mathcal{N}=4$ super-Yang-Mills theory. Hence no further hidden cancellations are present at three loops. We found that the divergence has a uniform degree of transcendentality, and is proportional to $\zeta_{3}$. We also evaluated the divergence of the three-loop four-graviton amplitude in 7, 9 and 11 dimensions; the latter results may be of interest in studies of $\mathrm{M}$ theory dualities [56]. 
While explicit calculations in $\mathcal{N}=8$ supergravity reveal cancellations beyond those needed for finiteness, their origin remains to be fully unraveled. For a subset of contributions, all-loop cancellations [11] follow from the "no-triangle" property at oneloop [4, 5, 6, 6, 8, 9, 10]. In ref. [21] one-loop cancellations in generic theories of gravity were linked to unexpectedly soft behavior of tree-level gravity amplitudes under large complex shifts of their momenta [24, 25, 26, 27, 28]. This mechanism was also proposed as a source of all-loop cancellations, which may be sufficiently strong to render the $\mathcal{N}=8$ theory finite, when combined with supersymmetric cancellations. This line of reasoning has been pursued further in ref. [10]. Improved ultraviolet properties in $\mathcal{N}=8$ supergravity have also been linked to $\mathrm{M}$ theory dualities [12, 15] and to string theory non-renormalization theorems [13, 14].

We also presented the fully color-dressed $\mathcal{N}=4$ super-Yang-Mills three-loop four-point amplitude. In our color decomposition the contact terms are given the same color factor as the parent diagram to which they are assigned. The parent diagrams contain only threevertices, each of which carries an $f^{a b c}$ color factor. The freedom to assign contact terms to different parent diagrams, and thereby to different color factors, cancels in the full amplitude. To confirm our color dressing for the three-loop four-point amplitudes, we evaluated the cuts using color-dressed tree amplitudes as input. We expect that, in general, any amplitude in any gauge theory can be color dressed by first assigning contact terms to parent diagrams, in a way that is consistent with all color-ordered unitarity cuts. Then one dresses the three-point vertices of the parent diagrams with the appropriate $f^{a b c}$ color factors.

Representations of amplitudes manifestly exhibiting all ultraviolet cancellations, such as the one presented in this paper, should be helpful for studying their properties and for tracking the origin of the cancellations, at both three and higher loop orders.

\section{Acknowledgments}

We thank David Kosower for many helpful discussions and collaboration on this topic. We also thank Paul Howe, Harald Ita, Renata Kallosh, Kelly Stelle and Pierre Vanhove for valuable discussions. We thank Academic Technology Services at UCLA for computer support. This research was supported by the US Department of Energy under contracts DE-FG03-91ER40662 (Z. B., J. J. M. C., H. J.), DE-AC02-76SF00515 (L. J. D.), DE- 
FG02-90ER40577 (OJI) (R. R.), the US National Science Foundation under grants PHY0455649 and PHY-0608114, and the A. P. Sloan Foundation (R. R.). J. J. M. C. and H. J. gratefully acknowledge the financial support of Guy Weyl Physics and Astronomy Alumni Fellowships.

[1] E. Cremmer, B. Julia and J. Scherk, Phys. Lett. B 76, 409 (1978);

E. Cremmer and B. Julia, Phys. Lett. B 80, 48 (1978); Nucl. Phys. B 159, 141 (1979).

[2] Z. Bern, J. J. Carrasco, L. J. Dixon, H. Johansson, D. A. Kosower and R. Roiban, Phys. Rev. Lett. 98, 161303 (2007) hep-th/0702112.

[3] Z. Bern, L. J. Dixon and D. A. Kosower, Nucl. Phys. B 513, 3 (1998) hep-ph/9708239; JHEP 0408, 012 (2004) hep-ph/0404293;

Z. Bern, V. Del Duca, L. J. Dixon and D. A. Kosower, Phys. Rev. D 71, 045006 (2005) hep-th/0410224].

[4] Z. Bern, L. J. Dixon, M. Perelstein and J. S. Rozowsky, Nucl. Phys. B 546, 423 (1999) hep-th/9811140].

[5] Z. Bern, N. E. J. Bjerrum-Bohr and D. C. Dunbar, JHEP 0505, 056 (2005) hep-th/0501137.

[6] N. E. J. Bjerrum-Bohr, D. C. Dunbar and H. Ita, Phys. Lett. B 621, 183 (2005) hep-th/0503102;

N. E. J. Bjerrum-Bohr, D. C. Dunbar, H. Ita, W. B. Perkins and K. Risager, JHEP 0612, 072 (2006) hep-th/0610043.

[7] R. Kallosh, 0711.2108 [hep-th].

[8] N. E. J. Bjerrum-Bohr and P. Vanhove, JHEP 0804, 065 (2008) [0802.0868 [hep-th]].

[9] N. E. J. Bjerrum-Bohr and P. Vanhove, 0805.3682 [hep-th]; 0806.1726 [hep-th].

[10] N. Arkani-Hamed, F. Cachazo and J. Kaplan, 0808.1446 [hep-th].

[11] Z. Bern, L. J. Dixon and R. Roiban, Phys. Lett. B 644, 265 (2007) hep-th/0611086].

[12] G. Chalmers, hep-th/0008162;

M. B. Green, J. G. Russo and P. Vanhove, JHEP 0702, 099 (2007) hep-th/0610299.

[13] N. Berkovits, Phys. Rev. Lett. 98, 211601 (2007) hep-th/0609006.

[14] M. B. Green, J. G. Russo and P. Vanhove, Phys. Rev. Lett. 98, 131602 (2007) hep-th/0611273]. 
[15] M. B. Green, H. Ooguri and J. H. Schwarz, Phys. Rev. Lett. 99, 041601 (2007) [0704.0777 [hep-th]].

[16] M. T. Grisaru, Phys. Lett. B 66, 75 (1977);

E. Tomboulis, Phys. Lett. B 67, 417 (1977);

S. Deser, J. H. Kay and K. S. Stelle, Phys. Rev. Lett. 38, 527 (1977);

P. S. Howe and K. S. Stelle, Int. J. Mod. Phys. A 4, 1871 (1989);

N. Marcus and A. Sagnotti, Nucl. Phys. B 256, 77 (1985).

[17] P. S. Howe and K. S. Stelle, Phys. Lett. B 554, 190 (2003) hep-th/0211279.

[18] M. T. Grisaru and W. Siegel, Nucl. Phys. B 201, 292 (1982) [Erratum-ibid. B 206, 496 (1982)].

[19] P. S. Howe and U. Lindstrom, Nucl. Phys. B 181, 487 (1981);

R. E. Kallosh, Phys. Lett. B 99, 122 (1981).

[20] K. S. Stelle, talk presented at the UCLA workshop: Is $\mathcal{N}=8$ Supergravity Finite?, http://www.physics.ucla.edu/tep/〜 workshops/supergravity/agenda/Talks/talk2.pdf

[21] Z. Bern, J. J. Carrasco, D. Forde, H. Ita and H. Johansson, Phys. Rev. D 77, 025010 (2008) [0707.1035 [hep-th]].

[22] R. Britto, F. Cachazo and B. Feng, Nucl. Phys. B 715, 499 (2005) hep-th/0412308.

[23] R. Britto, F. Cachazo, B. Feng and E. Witten, Phys. Rev. Lett. 94, 181602 (2005) hep-th/0501052.

[24] J. Bedford, A. Brandhuber, B. J. Spence and G. Travaglini, Nucl. Phys. B 721, 98 (2005) hep-th/0502146;

F. Cachazo and P. Svrček, hep-th/0502160;

N. E. J. Bjerrum-Bohr, D. C. Dunbar, H. Ita, W. B. Perkins and K. Risager, JHEP 0601, 009 (2006) hep-th/0509016;

A. Brandhuber, S. McNamara, B. Spence and G. Travaglini, JHEP 0703, 029 (2007) hep-th/0701187.

[25] P. Benincasa, C. Boucher-Veronneau and F. Cachazo, JHEP 0711, 057 (2007) hep-th/0702032;

P. Benincasa and F. Cachazo, 0705.4305 [hep-th];

A. Hall, Phys. Rev. D 77, 124004 (2008) [0803.0215 [hep-th]].

[26] H. Elvang, D. Z. Freedman and M. Kiermaier, 0808.1720 [hep-th].

[27] N. Arkani-Hamed and J. Kaplan, JHEP 0804, 076 (2008) [0801.2385 [hep-th]]. 
[28] C. Cheung, 0808.0504 [hep-th].

[29] G. Chalmers and W. Siegel, Phys. Rev. D 59, 045013 (1999) hep-ph/9801220;

D. Vaman and Y. P. Yao, JHEP 0604, 030 (2006) hep-th/0512031]; 0805.2645 [hep-th].

[30] G. 't Hooft and M. J. G. Veltman, Annales Poincare Phys. Theor. A 20, 69 (1974).

[31] S. Deser and P. van Nieuwenhuizen, Phys. Rev. D 10, 401 (1974);

S. Deser, H. S. Tsao and P. van Nieuwenhuizen, Phys. Rev. D 10, 3337 (1974).

[32] D. C. Dunbar and P. S. Norridge, Class. Quant. Grav. 14, 351 (1997) hep-th/9512084;

D. C. Dunbar and P. S. Norridge, Nucl. Phys. B 433, 181 (1995) hep-th/9408014].

[33] R. E. Kallosh, Nucl. Phys. B 78, 293 (1974).

[34] P. van Nieuwenhuizen and C. C. Wu, J. Math. Phys. 18, 182 (1977).

[35] M. H. Goroff and A. Sagnotti, Phys. Lett. B 160, 81 (1985); Nucl. Phys. B 266, 709 (1986).

[36] A. E. M. van de Ven, Nucl. Phys. B 378, 309 (1992).

[37] Z. Bern, L. J. Dixon, D. C. Dunbar and D. A. Kosower, Nucl. Phys. B 425, 217 (1994) hep-ph/9403226;

Z. Bern, L. J. Dixon, D. C. Dunbar and D. A. Kosower, Nucl. Phys. B 435, 59 (1995) hep-ph/9409265.

[38] Z. Bern, L. J. Dixon, D. C. Dunbar, M. Perelstein and J. S. Rozowsky, Nucl. Phys. B 530, 401 (1998) hep-th/9802162.

[39] Z. Bern, J. S. Rozowsky and B. Yan, Phys. Lett. B 401, 273 (1997) hep-ph/9702424.

[40] H. Kawai, D. C. Lewellen and S. H. H. Tye, Nucl. Phys. B 269, 1 (1986).

[41] Z. Bern and A. K. Grant, Phys. Lett. B 457, 23 (1999) hep-th/9904026;

Z. Bern, A. De Freitas and H. L. Wong, Phys. Rev. Lett. 84, 3531 (2000) hep-th/9912033;

N. E. J. Bjerrum-Bohr, Phys. Lett. B 560, 98 (2003) hep-th/0302131; Nucl. Phys. B 673, 41 (2003) hep-th/0305062;

N. E. J. Bjerrum-Bohr and K. Risager, Phys. Rev. D 70, 086011 (2004) hep-th/0407085;

S. Ananth and S. Theisen, Phys. Lett. B 652, 128 (2007) [0706.1778 [hep-th]];

H. Elvang and D. Z. Freedman, JHEP 0805, 096 (2008) [0710.1270 [hep-th]].

[42] Z. Bern, Living Rev. Rel. 5, 5 (2002) gr-qc/0206071.

[43] M. Bianchi, H. Elvang and D. Z. Freedman, 0805.0757 [hep-th].

[44] Z. Bern, J. J. M. Carrasco and H. Johansson, 0805.3993 [hep-ph].

[45] Z. Bern, J. J. M. Carrasco, H. Johansson and D. A. Kosower, Phys. Rev. D 76, 125020 (2007) 
[0705.1864 [hep-th]].

[46] R. Britto, F. Cachazo and B. Feng, Nucl. Phys. B 725, 275 (2005) hep-th/0412103;

E. I. Buchbinder and F. Cachazo, JHEP 0511, 036 (2005) hep-th/0506126.

[47] E. Witten, Commun. Math. Phys. 252, 189 (2004) hep-th/0312171.

[48] F. Cachazo and D. Skinner, 0801.4574 [hep-th];

F. Cachazo, 0803.1988 [hep-th].

[49] F. Cachazo, M. Spradlin and A. Volovich, 0805.4832 [hep-th];

M. Spradlin, A. Volovich and C. Wen, 0808.1054 [hep-th].

[50] Z. Bern and D. A. Kosower, Nucl. Phys. B 379, 451 (1992);

Z. Bern, A. De Freitas, L. J. Dixon and H. L. Wong, Phys. Rev. D 66, 085002 (2002) hep-ph/0202271.

[51] W. Siegel, Phys. Lett. B 84, 193 (1979).

[52] V. P. Nair, Phys. Lett. B 214, 215 (1988).

[53] E. Witten, Commun. Math. Phys. 252, 189 (2004) hep-th/0312171.

[54] G. Georgiou, E. W. N. Glover and V. V. Khoze, JHEP 0407, 048 (2004) hep-th/0407027.

[55] J. M. Drummond, J. Henn, G. P. Korchemsky and E. Sokatchev, 0807.1095 [hep-th];

A. Brandhuber, P. Heslop and G. Travaglini, 0807.4097 [hep-th];

J. M. Drummond, J. Henn, G. P. Korchemsky and E. Sokatchev, 0808.0491 [hep-th];

J. M. Drummond and J. M. Henn, 0808.2475 [hep-th].

[56] M. B. Green, M. Gutperle and P. Vanhove, Phys. Lett. B 409, 177 (1997) hep-th/9706175;

M. B. Green, H. h. Kwon and P. Vanhove, Phys. Rev. D 61, 104010 (2000) hep-th/9910055;

M. B. Green and P. Vanhove, JHEP 0601, 093 (2006) hep-th/0510027;

M. B. Green, J. G. Russo and P. Vanhove, JHEP 0802, 020 (2008) [0801.0322 [hep-th]]; JHEP

0807, 126 (2008) [0807.0389 [hep-th]].

[57] R. Akhoury, Phys. Rev. D 19, 1250 (1979);

A. H. Mueller, Phys. Rev. D 20, 2037 (1979);

J. C. Collins, Phys. Rev. D 22, 1478 (1980);

A. Sen, Phys. Rev. D 24, 3281 (1981);

G. Sterman, Nucl. Phys. B 281, 310 (1987);

J. Botts and G. Sterman, Phys. Lett. B 224, 201 (1989) [Erratum-ibid. B 227, 501 (1989)];

S. Catani and L. Trentadue, Nucl. Phys. B 327, 323 (1989); 
G. P. Korchemsky, Phys. Lett. B 220, 629 (1989);

L. Magnea and G. Sterman, Phys. Rev. D 42, 4222 (1990);

G. P. Korchemsky and G. Marchesini, Phys. Lett. B 313 (1993) 433;

S. Catani, Phys. Lett. B 427, 161 (1998) hep-ph/9802439];

G. Sterman and M. E. Tejeda-Yeomans, Phys. Lett. B 552, 48 (2003) hep-ph/0210130;

L. J. Dixon, L. Magnea and G. Sterman, JHEP 0808, 022 (2008) [0805.3515 [hep-ph]].

[58] A. Sen, Phys. Rev. D 28, 860 (1983);

J. Botts and G. Sterman, Nucl. Phys. B 325, 62 (1989);

N. Kidonakis, G. Oderda and G. Sterman, Nucl. Phys. B 525, 299 (1998) hep-ph/9801268];

Nucl. Phys. B 531, 365 (1998) hep-ph/9803241;

A. Banfi, G. P. Salam and G. Zanderighi, JHEP 0408, 062 (2004) hep-ph/0407287.

[59] S. Mert Aybat, L. J. Dixon and G. Sterman, Phys. Rev. Lett. 97, 072001 (2006) hep-ph/0606254; Phys. Rev. D 74, 074004 (2006) hep-ph/0607309.

[60] G. P. Korchemsky and A. V. Radyushkin, Phys. Lett. B 171 (1986) 459;

S. V. Ivanov, G. P. Korchemsky and A. V. Radyushkin, Yad. Fiz. 44 (1986) 230 [Sov. J. Nucl. Phys. 44 (1986) 145].

[61] Z. Bern, L. J. Dixon and V. A. Smirnov, Phys. Rev. D 72, 085001 (2005) hep-th/0505205.

[62] V. A. Smirnov, Phys. Lett. B 567, 193 (2003) hep-ph/0305142.

[63] C. Anastasiou, Z. Bern, L. J. Dixon and D. A. Kosower, Phys. Rev. Lett. 91, 251602 (2003) hep-th/0309040.

[64] F. Cachazo, M. Spradlin and A. Volovich, Phys. Rev. D 74, 045020 (2006) hep-th/0602228;

Z. Bern, M. Czakon, D. A. Kosower, R. Roiban and V. A. Smirnov, Phys. Rev. Lett. 97, 181601 (2006) hep-th/0604074;

L. F. Alday and J. Maldacena, JHEP 0706, 064 (2007) [0705.0303 [hep-th]]; JHEP 0711, 068 (2007) [0710.1060 [hep-th]];

J. M. Drummond, J. Henn, G. P. Korchemsky and E. Sokatchev, 0712.1223 [hep-th];

J. Bartels, L. N. Lipatov and A. S. Vera, 0802.2065 [hep-th]; 0807.0894 [hep-th];

Z. Bern, L. J. Dixon, D. A. Kosower, R. Roiban, M. Spradlin, C. Vergu and A. Volovich, Phys. Rev. D 78, 045007 (2008) [0803.1465 [hep-th]].

[65] S. Weinberg, Phys. Rev. 140, B516 (1965).

[66] S. G. Naculich, H. Nastase and H. J. Schnitzer, 0805.2347 [hep-th]; 
A. Brandhuber, P. Heslop, A. Nasti, B. Spence and G. Travaglini, 0805.2763 [hep-th].

[67] M. K. Gaillard and B. Zumino, Nucl. Phys. B 193, 221 (1981);

B. de Wit and H. Nicolai, Nucl. Phys. B 208, 323 (1982).

[68] L. Brink, S. S. Kim and P. Ramond, JHEP 0806, 034 (2008) [0801.2993 [hep-th]];

R. Kallosh and M. Soroush, Nucl. Phys. B 801, 25 (2008) [0802.4106 [hep-th]].

[69] P. S. Howe and K. S. Stelle, Phys. Lett. B 137, 175 (1984).

[70] A. Galperin, E. Ivanov, S. Kalitsyn, V. Ogievetsky and E. Sokatchev, Class. Quant. Grav. 1, 469 (1984); Class. Quant. Grav. 2, 155 (1985).

[71] Z. Bern and A. G. Morgan, Nucl. Phys. B 467, 479 (1996) hep-ph/9511336; Z. Bern, L. J. Dixon and D. A. Kosower, Ann. Rev. Nucl. Part. Sci. 46, 109 (1996) hep-ph/9602280; Z. Bern, L. J. Dixon, D. C. Dunbar and D. A. Kosower, Phys. Lett. B 394, 105 (1997) hep-th/9611127;

Z. Bern, L. J. Dixon and D. A. Kosower, JHEP 0001, 027 (2000) hep-ph/0001001].

[72] F. A. Berends, R. Kleiss, P. De Causmaecker, R. Gastmans and T. T. Wu, Phys. Lett. B 103, 124 (1981);

P. De Causmaecker, R. Gastmans, W. Troost and T. T. Wu, Nucl. Phys. B 206, 53 (1982);

Z. Xu, D. H. Zhang and L. Chang, TUTP-84/3-TSINGHUA;

R. Kleiss and W. J. Stirling, Nucl. Phys. B 262, 235 (1985);

J. F. Gunion and Z. Kunszt, Phys. Lett. B 161, 333 (1985);

Z. Xu, D. H. Zhang and L. Chang, Nucl. Phys. B 291, 392 (1987).

[73] V. Del Duca, A. Frizzo and F. Maltoni, Nucl. Phys. B568, 211 (2000) hep-ph/9909464;

V. Del Duca, L. J. Dixon and F. Maltoni, Nucl. Phys. B 571, 51 (2000) hep-ph/9910563.

[74] R. Kleiss and H. Kuijf, Nucl. Phys. B 312, 616 (1989).

[75] A. G. Grozin, Int. J. Mod. Phys. A 19, 473 (2004) hep-ph/0307297.

[76] F.V. Tkachov, Phys. Lett. B 100, 65 (1981);

K.G. Chetyrkin and F.V. Tkachov, Nucl. Phys. B 192, 159 (1981). 